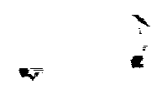

NASA Technical Memorandum 101724

\section{IN \\ Od}

1635

p.36

\title{
F-18 High Alpha Research Vehicle Surface Pressures: Initial In-Flight Results and Correlation With Flow Visualization and Wind-Tunnel Data
}

David F. Fisher, Daniel W. Banks, and David M. Richwine

(NASA-TM-1017?4) F-18 HIGH ALPHA RESEARCH VFHICLE SURFACE PRESSURFS: INITIAL IN-FLIOHT RESULTS AND CORRELATION WITH FLOW VISUALIZATION AND WIND-TUNNEL DATA $36 \mathrm{p}$
N91-19051

(NASA) CSCL OIA GJ/O?
Unclas

0001635

August 1990

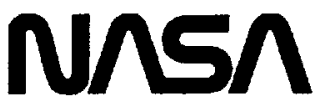

National Aeronautics and

Space Administration 
Ames Research Center

Dryden Flight Research Facility

P.O. Box 273

Edwards, California 93523-0273

TO: $\quad$ Report Recipient

FROM: $\quad$ ATD/Acting Chief, Dryden Reports \& Presentations Branch

SUBJECT: Report Errata

Figure 10(a) (fuselage station 142) of NASA Technical Memorandum 101724 has been corrected. Please substitute page 6 in the text portion (second column, last two paragraphs contain revised material) and page 23 in the figures section of the report with the enclosed corrections.

marilee ti marean

Marilee S. Morgan

Enclosures 


\section{ERRATA}

NASA Technical Memorandum 101724

F-18 High Alpha Research Vehicle Surface Pressures: Initial In-Flight Results and Correlation With Flow Visualization and Wind-Tunnel Data

David F. Fisher, Daniel W, Banks, and David M. Richwine

August 1990

Figure 10(a) (fuselage station 142) of NASA Technical Memorandum 101724 has been corrected. Please substitute page 6 in the text portion (second column, last two paragraphs contain revised material) and substitute page 23 in the figures section of the report with the enclosed corrections. 

NASA Technical Memorandum 101724

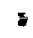

: F-18 High Alpha Research Vehicle Surface Pressures: Initial In-Flight Results and Correlation With Flow Visualization and Wind-Tunnel Data

David F. Fisher

NASA Ames Research Center, Dryden Flight Research Facility, Edwards, California

Daniel W. Banks

NASA Langley Research Center, Hampton, Virginia

David M. Richwine

PRC Systems Services, Edwards, California

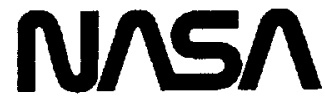

National Aeronautics and

Space Administration

Ames Research Center

Dryden Flight Research Facility

Edwards, California 93523-0273 


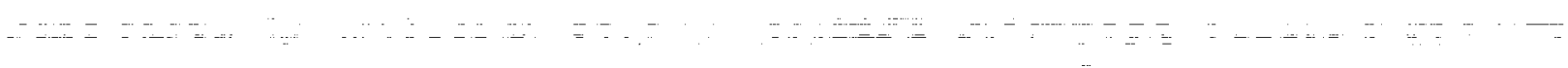

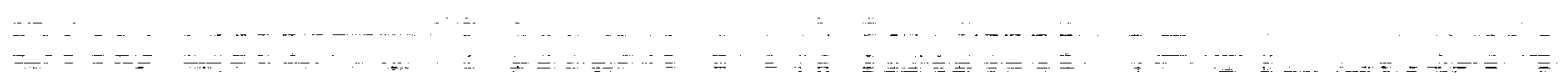




\section{F-18 HIGH ALPHA RESEARCH VEHICLE SURFACE PRESSURES: INITIAL IN-FLIGHT RESULTS AND CORRELATION WITH FLOW VISUALIZATION AND WIND-TUNNEL DATA}

\author{
David F. Fisher* \\ NASA Ames Research Center \\ Dryden Flight Research Facility \\ Edwards, CA
}

\author{
Danicl W. Banks* \\ NASA Langley Research Center \\ Hampton, VA
}

\author{
David M. Richwine* \\ PRC Systems Services \\ Edwards, CA
}

\begin{abstract}
Pressure distributions have been reported at angles of attack from $10^{\circ}$ to $50^{\circ}$ and at Mach 0.23 to 0.60 at five fuselage stations on the forebody and at three fuselage stations on the leading-edge extensions of the NASA F-18 high alpha research vehicle (HARV). The reported results have been correlated with flow visualization results obtained during a previous investigation on the HARV and with test results obtained from a 6-percent-scale F-18 wind-tunnel model. A general trend in the data from the forebody was for the maximum suction pressure peaks to first appear at angle of attack $(\alpha) \sim 19^{\circ}$ and increase in magnitude as angle of attack was increased. The general trend of the leadingedge extension (LEX) pressure distribution was the inward progression of the maximum suction peaks, the increase in magnitude of the maximum suction pressure pcaks up to vortex core breakdown, and then the decrease and general flattening of the pressure distribution beyond the LEX primary vortex core breakdown. No significant effect of Mach number was noted for the forebody results for the Mach number range reported. However, at all three LEX orifice stations a substantial compressibility effect resulted in a significant reduction in vortex-induced suction pressure as Mach number increased. The forebody primary and the LEX secondary vortex separation lines, as identified by the surface flow visualization, correlated well with the end

-Aerospace Engineer. Member AIAA.

Copyright (C) 1990 by the American Institute of Aeronautics and Astronautics, Inc. No copyright is asserted in the United States under Title 17, U.S. Code. The U.S. Government has a royalty-free license to exercise all rights under the copyright claimed herein for Governmental purposes. All other rights are reserved by the copyright owner.
\end{abstract}

of pressure recovery, leeward and windward respectively, of the maximum suction pressure peaks. The flight to wind-tunnel correlations were generally good, with some exceptions.

\begin{tabular}{|c|c|}
\hline & Nomenclature \\
\hline$C_{\mathrm{p}}$ & pressure coefficient \\
\hline$C_{p}^{*}$ & $\begin{array}{l}\text { pressure coefficient corresponding } \\
\text { to local speed of sound, } \\
C_{p}^{*}=\left(\frac{2}{\gamma M_{\infty}^{2}}\right)\left\{\left(\frac{(\gamma-1) M_{\infty}^{2}+2}{\gamma+1}\right)^{3.5}-1\right\}\end{array}$ \\
\hline CFD & computational fluid dynamics \\
\hline CRT & cathode ray tube \\
\hline$d$ & diameter of fuselage forebody, in. \\
\hline$d_{\max }$ & $\begin{array}{l}\text { diameter of fuselage forebody along } \\
\text { major axis, in. }\end{array}$ \\
\hline$d_{\min }$ & $\begin{array}{l}\text { diameter of fuselage forebody along } \\
\text { minor axis, in. }\end{array}$ \\
\hline ECM & electronic counter measures \\
\hline F.S. & $\begin{array}{l}\text { fuselage station, in. (nose apex at } \\
59.82 \mathrm{in} \text {.) }\end{array}$ \\
\hline HARV & high alpha research vehicle \\
\hline HATP & High Alpha Technology Program \\
\hline LEX & leading-edge extension \\
\hline LSB & laminar separation bubble \\
\hline$\ell$ & $\begin{array}{l}\text { length of aircraft from nose apex to } \\
\text { engine exhaust plane, } 54.4 \mathrm{ft}\end{array}$ \\
\hline$M_{\infty}$ & free-stream Mach number \\
\hline m.a.c. & mean aerodynamic chord, $11.525 \mathrm{ft}$ \\
\hline $\mathrm{PCM}$ & pulse code modulation \\
\hline
\end{tabular}




\begin{tabular}{|c|c|}
\hline PROM & programmed read only memory \\
\hline$R$ & reattachment line location \\
\hline$R e_{\bar{c}}$ & $\begin{array}{l}\text { Reynolds number based on mean } \\
\text { aerodynamic chord }\end{array}$ \\
\hline$R e_{d}$ & Reynolds number based on diameter \\
\hline$R e_{d^{\prime}}$ & $\begin{array}{l}\text { Reynolds number based on local } \\
\text { maximum fuselage diameter, corrected } \\
\text { for angle of attack using the method of } \\
\text { Ref. } 17\end{array}$ \\
\hline$S_{1}$ & primary separation line location \\
\hline$S_{2}$ & secondary separation line location \\
\hline$S_{3}$ & tertiary separation line location \\
\hline$s$ & $\begin{array}{l}\text { local span distance from LEX fuselage } \\
\text { junction to LEX leading edge, in. }\end{array}$ \\
\hline$x$ & $\begin{array}{l}\text { distance from nose apex along longitudinal } \\
\text { axis of aircraft, } \mathrm{ft}\end{array}$ \\
\hline$y$ & distance along LEX local semispan, in. \\
\hline$\alpha$ & $\begin{array}{l}\text { aircraft angle of attack, deg, right wingtip } \\
\text { angle-of-attack vane corrected for } \\
\text { upwash and boom bending }\end{array}$ \\
\hline$\alpha^{\prime}$ & $\begin{array}{l}\text { angle of attack used in the determination } \\
\text { of } R e_{d^{\prime}} \text { (at F.S. } 70,85 \text {, and } 107 \text {, } \\
\alpha^{\prime}=\alpha-5.6^{\circ} \text { because the nosecone } \\
\text { is depressed from the horizon } 5.6^{\circ} \text {; } \\
\text { at F.S. } 142 \text { and } 184, \alpha^{\prime}=\alpha \text { ) }\end{array}$ \\
\hline$\beta$ & $\begin{array}{l}\text { aircraft angle of sideslip, deg, average of } \\
\text { left- and right-wingtip sideslip vanes } \\
\text { corrected for angle of attack }\end{array}$ \\
\hline$\Delta$ & differential \\
\hline$\gamma$ & ratio for specific heats of air, 1.4 \\
\hline$\theta$ & $\begin{array}{l}\text { forcbody cross-section circumferential } \\
\text { angle, deg, }\left(0^{\circ} \text { is bottom centerline, }\right. \\
\text { positive is clockwise as seen from a } \\
\left.\text { front view, } 0^{\circ} \text { to } 360^{\circ}\right)\end{array}$ \\
\hline
\end{tabular}

\section{Introduction}

In recent years, more emphasis has been placed on expanding the envelope of fighter aircraft to include controlled flight at high angle of attack. Fighters such as the F-18 and F-16 aircraft utilize leading-edge extensions (LEXs) or wing body strakes which provide additional lift because of the vortical flow these devices develop at moderate-to-high angles of attack. ${ }^{1}$ However, the prediction and control of this vortical flow and the mutual interactions of the vortices are not well understood. The combined effect of the LEX or wing body strake vortices, as well as the forebody vortices on the vehicle aerodynamics, must be considered to avoid any adverse stability and control and other problems such as buffet.

Understanding the vortical flow interactions on scale models in wind tunnels can be difficult. Experimental tests in wind tunnels with different scale models have provided conflicting results, even with tests conducted at the same Reynolds number. ${ }^{2}$ In such subscale model tests, the interaction of the forebody and LEX vortices on 6- and 7-percent scale F-18 models typically resulted in apparent lateral stability for all angles of attack, including stall and poststall regions. However, airplane flight data and wind-tunnel test results for the large-scale (16 percent) model, at low Reynolds numbers, indicated a region of lateral instability near maximum lift. This apparent scale effect still has not been resolved. Understanding such scale effects is essential for the successful design of future fighters intended to operate at high angles of attack.

Currently NASA is conducting a High Alpha Technology Program (HATP) to increase the understanding, improve prediction techniques, provide design guidelines, and investigate new concepts for vortex control on advanced, highly maneuverable aircraft at high angles of attack. This program utilizes the F-18 configuration as a validation and demonstration approach. It consists of wind-tunnel tests of subscale ${ }^{3-4}$ and full-scale models and components; calibration for computational fluid dynamics (CFD) $\operatorname{codes}^{5-9}$ piloted simulations, and full-scale flight testing. ${ }^{10-16}$ (Also: Fisher, D.F., Curry, R.E., Del Frate, J.H., and Richwine, D.M., paper to be published in the serial Flow Visualization $V$, and Fisher, David F., Del Frate, John H., and Richwine, David M., NASA Technical Memorandum, to be published.)

As part of this program, extensive pressure distribution and flow visualization studies have been conducted on the NASA F-18 high alpha research vehicle (HARV) at the Ames Research Center, Dryden Flight Research Facility. Surface and off-surface flow visualization results (obtained in-flight on the NASA F-18 HARV, highlighting the extensive vortical threedimensional separated flow on the aircraft at angles of attack up to $55^{\circ}$ ) have been reported in Refs. 10-15.

In this paper, results from pressure measurements on the forebody and the leading-edge extensions (LEX) are reported from both stabilized and dynamic flight conditions, at Mach 0.23 to 0.60 , and at Reynolds num- 
bers up to $25 \times 10^{6}$ based on wing mean aerodynamic chord (m.a.c.). Also presented are correlations with previously obtained results from flow visualization and from wind-tunnel pressure measurements on subscale models.

\section{Vehicle Description}

The NASA HARV (Fig. 1) is a single-place preproduction F-18 aircraft built by the McDonnell Douglas (St. Louis, Missouri) and Northrop (Newbury Park, Califomia) corporations. It is powered by two GE (General Electric, Lynn, Massachusetts) F404-GE-400 afterbuming turbofan engines. The aircraft features a midwing with leading- and trailing-edge flaps which operate on a schedule that is a function of angle of attack and Mach number. For free-stream Mach number $\left(M_{\infty}\right) \leq 0.76$ and angle of attack $(\alpha) \geq 26^{\circ}$, the leading-edge flap is down $34^{\circ}$ (maximum), and the trailing-edge flap is at $0^{\circ}$. Leading-edge extensions are mounted on each side of the fuselage from the wing roots to just forward of the windscreen. The aircraft has twin vertical stabilizers canted out $20^{\circ}$ from the vertical and differential all-moving horizontal tails.

The NASA F-18 HARV, with the current flight control computers and control laws (8.3.3 programmed read only memory (PROM) set), is flown by NASA pilots in the fighter escort configuration without stores. The aircraft carries no missiles and the wingtip Sidewinder missile launch racks have been replaced with special camera pods and wingtip airdata booms. The flight test noseboom has been removed from the aircraft and a NASA flush airdata system ${ }^{16}$ has been installed. The aircraft has an unrestricted angle-ofattack flight envelope in this configuration, with the center of gravity between 17 percent and 25 percent m.a.c., as defined by the Naval Air Training and Operating Procedures Standardization (NATOPS) manual.

\section{Experiment Description}

Pressure measurements were made on both the forebody and the LEXs of the F-18 HARV at selected fuselage locations. These fuselage locations correspond with orifice locations on both the 6- and 16-percent scale models.

Five rings of static pressure orifices were installed on the fuselage forward of the canopy as shown in Fig. 2. At the first 2 rows, fuselage station (F.S.) 70 (ratio of length from apex to fuselage length $(x / \ell)=0.015)$ and F.S. $85(x / \ell=0.038), 30$ static pressure orifices were equally spaced about the nosecone. Two extra orifices were located at the forebody cross-section circumferential angle $(\theta)=90^{\circ}$ and $270^{\circ}\left(0^{\circ}\right.$ is bottom centerline, positive is clockwise as seen from a front view, $0^{\circ}$ to $360^{\circ}$ ). At the last 3 rows, F.S. $107(x / \ell=0.071)$, F.S. 142 $(x / \ell=0.126)$, and F.S. $184(x / \ell=0.190) 64$ orifices were spaced about the fuselage at each location with orifices more closely spaced (every $3^{\circ}$ ) on the upper surface where larger pressure gradients were expected. At F.S. 184, two large vents for the gun bay were located on the lower portion of the fuselage and no orifices were installed there.

Both the left and right LEXs were instrumented with three rows of pressure orifices located at F.S. 253 $(x / \ell=0.295)$, F.S. $296(x / \ell=0.361)$, and F.S. 357 $(x / \ell=0.454)$. Approximately 13 to 20 orifices were installed at each station on the upper surface of each LEX and 4 to 5 at each station on the lower surface, respectively. The orifices were located with the closest spacing near the leading edge. A total of 384 surface static pressure measurements were made on the fuselage and LEXs.

A cross section of the orifice stations and the orientation of the orifices is given in Fig. 3. The view is looking aft on the aircraft with the bottom fuselage centerline at $0^{\circ}$ and the top centerline fuselage at $180^{\circ}$. The first three fuselage stations were circular in cross section and were canted forward $5.6^{\circ}$ to coincide with the depression of the nosecone centerline. At F.S. 142 and F.S. 184, the fuselage cross section became somewhat elliptical with the major axis in the vertical plane. The ratio of the major to minor axis was 1.10 and 1.35 for F.S. 142 and F.S. 184, respectively. At the LEX stations, $y / s=0.0$ is defined as the LEX fuselage junction while $y / s=1.0$ is the leading edge of the LEX, +1.0 for the left-LEX leading edge and -1.0 for the rightLEX lcading edge.

Several protrusions and discontinuities on the fuselage should be noted. The nosecone was generally smooth and free of discontinuities, with the noseconefuselage junction at F.S. 128.5. Two small ellipticalshaped electronic counter measures (ECM) antenna covers, Fig. 4(a), were located on the sides of the fuselage centered at F.S. $134, \theta=85^{\circ}$ and $275^{\circ}$ and were approximately $9.5 \mathrm{in}$. long, 4 in. wide and protruded approximately 1.7 in. Located on the upper surface, between F.S. 128.5 and F.S. 188 and between $\theta \sim$ $138^{\circ}$ and $165^{\circ}$, were doors covering the in-flight refueling probe. While these doors were nominally flush, there were gaps and small protrusions present. Two 
small production angle-of-attack vanes were installed at F.S. 165, one at $\theta \sim 70^{\circ}$ and the other at $\theta \sim 290^{\circ}$. Two aircraft production pitot-static probes, Fig. 4(a), were located on the lower fuselage at F.S. 164 to F.S. 177 and $\theta=35^{\circ}$ and $325^{\circ}$. On this preproduction aircraft, the gun port exits normally located on the upper centerline near F.S. 122, were replaced by a fairing without ports. Of the protrusions noted, the ECM antenna covers and the production pitot-static probes had the greatest effect on the pressure distributions, as will be shown later. The LEX, Fig. 4(b), was virtually free of significant protrusions forward of the orifice rows.

\section{Instrumentation}

Each orifice on the forebody was connected to temperature-controlled electronic scanning pressure modules with $6 \mathrm{ft}$ of 0.062 -in. id pneumatic tubing. On the LEXs, the tubing for each orifice was matched in length at each orifice row but varied from $21 / 2$ to $31 / 2 \mathrm{ft}$ at the three stations. It was previously determined that $8 \mathrm{ft}$ of 0.062 -in. flexible tubing would have a pneumatic lag of approximately $10 \mathrm{msec}$ at an altitude of $20,000 \mathrm{ft}^{16}$ Reference pressure for the modules was supplied through $1 / 4$-in. tubing to a reference pressure tank with an intermal volume of $50 \mathrm{in}^{3}$ located in the forebody, vented to the radome compartment, and monitored by a high-resolution absolute pressure transducer. The pressure transducer within each module was scanned sequentially 25 times/sec by a 10-bit pulse code modulation (PCM) data system. In-flight zero differential pressure readings were taken before each test point and were used during postflight data reduction to correct the data for calibration offsets. On the forebody, $\pm 216 \mathrm{lb} / \mathrm{ft}^{2}$ differential range transducers were used while $\pm 720 \mathrm{lb} / \mathrm{ft}^{2}$ differential range transducers were used on the LEXs. Accuracy for the forebody pressure measurements is estimated to be $1 \mathrm{lb} / \mathrm{ft}^{2}$ and $3.5 \mathrm{lb} / \mathrm{ft}^{2}$ for the LEX measurements.

Airspeed, altitude, angle of attack, and angle of sideslip were measured using airspeed booms mounted on specially designed wingtip photo pods as shown in Fig. 5. On the right wingtip, Fig. 5(a), a standard NACA noseboom ${ }^{18}$ was installed with the tip mounted $7.3 \mathrm{ft}$ forward of the wingtip lcading edgc. On the left wingtip a swiveling probe, Fig. 5(b), was similarly located. The swiveling probe was designed with four vanes to align the probe head with the local airstream. The probe could align with the airstream for aircraft angles of attack from $-10^{\circ}$ to $+50^{\circ}$ and angles of sideslip greater than $\pm 20^{\circ}$. Free-stream Mach number, altitude, and dynamic pressure were determined using calibrated data from the swiveling probe on the left wingboom. Aircraft angle of attack was measured by using a vane on the right wingboom and corrected for upwash and boom bending. Angle of sideslip was determined as the average of the left-and right-wingboom sideslip vane measurement corrected for angle of attack. From unpublished data, it is estimated that angle of attack and angle of sideslip were accurate to $\pm 0.5^{\circ}$ for angles of attack up to $40^{\circ}$, and $\pm 1^{\circ}$ for angles of attack up to $50^{\circ}$. It is also estimated that $M_{\infty}$ is accurate to \pm 0.005 at $\alpha=50^{\circ}$, and \pm 0.003 for angles of attack below $30^{\circ}$.

The data from the above measurements, as well as the standard aircraft control positions, inertial system, and accelerometer parameters were transmitted to a ground station. These critical parameters were monitored by engineers and technicians in real time on strip charts, cathode ray tube (CRT) displays, and pressure distribution plots.

\section{Flight Test Conditions}

Data were obtained in both quasi-stabilized 1-g flight mancuvers, as well as windup tums and spiral dives. Data were obtained at nominal altitudes of 20,000 and $45,000 \mathrm{ft}$. At the higher angles of attack, constant altitude could not be maintained during the l-g maneuvers and data were obtained in a descent. During the windup tums and spiral dives the maneuvers were much more transient. In the first case the Mach number decreased rapidly. In the second case the altitude decreased rapidly. As mentioned previously in the Instrumentation section, the frequency response of the pressure system was less than $10 \mathrm{msec}$ and little lag was introduced. Time segments of $0.4-\mathrm{sec}$ duration were used for data analysis purposes, with approximately 10 time-points averaged.

\section{Results and Discussion}

\section{Forebody Results}

The forebody pressure distribution results are presented as pressure coefficients as a function of circumferential angle, $(\theta)$ for each of the five forebody stations. The effect of angle of attack and Mach number, as well as the correlation with flow visualization and wind-tunnel test results, are presented in the following sections. 


\section{Effect of Angle of Attack}

The effects of angle of attack on the forebody pressure coefficients are presented in Fig. 6 for angles of attack from $10^{\circ}$ to $50^{\circ}$. These data are at low speed $\left(0.23<M_{\infty}<0.40\right)$ and $1-g$ flight conditions. At F.S. 70, F.S. 85, and F.S. 107 and starting at $\alpha=19.7^{\circ}$, Fig. 6(a), the flow accelerated around the forebody and induced a pair of maximum suction pressure peaks on the side of the fuselage at $\theta \sim 120^{\circ}$ and $240^{\circ}$. As the angle of attack was increased, these maximum suction peaks became much more pronounced and moved down around the fuselage to $\theta \sim 95^{\circ}$ to $100^{\circ}\left(\sim 260^{\circ}\right.$ to $265^{\circ}$ ) at $\alpha=50^{\circ}$, Fig. $6($ c). At F.S. 85 and F.S. 107 , "footprints" 4 of the forebody primary vortex pair are indicated by suction pressure peaks at $\theta=168^{\circ}$ and $192^{\circ}$ which started at $\alpha=34.3^{\circ}$, Fig. 6(b), and became more negative as the angle of attack was increased. These footprints or suction peaks indicate the presence of the primary vortex cores above the surface. ${ }^{19}$ It should be noted that at these fuselage stations the pressure distributions in all cases are very symmetrical for $\beta \sim 0^{\circ}$, for angles of attack up to $50^{\circ}$.

At F.S. 142, sharp peaks at $\theta=90^{\circ}$ and $270^{\circ}$ in the pressure coefficient curve starting at $\alpha=19.7^{\circ}$, Fig. 6(a), are the result of local separation behind the two small elliptical-shaped ECM antenna covers described previously in the Experiment Description section. As the angle of attack is increased to $50^{\circ}$, Fig. 6(c), these peaks move up around the fuselage to $\theta=108^{\circ}$ and $252^{\circ}$.

At F.S. 142, the forebody cross section has become elliptical in shape. The maximum suction pressure peaks at this location have become flatter and the magnitude somewhat diminished above $\alpha \sim 25^{\circ}$ or $30^{\circ}$, Figs. $6(\mathrm{~b})$ and (c), compared to the three forward stations. Distinct footprints of the primary vortex are present near $\theta \sim 160^{\circ}$ and $200^{\circ}$, beginning around $\alpha=25^{\circ}$. Fig. 6(b). These footprint peaks are less distinct at $\theta \sim 160^{\circ}$ than at $\theta \sim 200^{\circ}$ because this area of the aircraft contains the doors for the in-flight refueling probe. This area is not as smooth and flush as the left side where there are no doors, joints, or other discontinuities. These footprint peaks reach the maximum level at $\alpha=45^{\circ}$, diminishing significantly in magnitude at $\alpha=50^{\circ}$, probably indicating that the vortices have begun to lift from the surface. Again, the pressure distributions are generally symmetrical with the differences accounted for by local protuberances or discontinuities.
At F.S. 184, the maximum suction pressure peaks have moved up to $\theta \sim 120^{\circ}$ and $240^{\circ}$, Figs. 6(b) and (c), because of the local influence of the LEX. At this station, the apex of the LEXs is only $13 \mathrm{in}$. aft, Fig. 4(a), and is located at $\theta \sim 123^{\circ}$ and $237^{\circ}$. As seen in Fig. 4(a), the surface streamlines are pulled up and over the LEX for $\alpha \sim 26^{\circ}$. The maximum suction peaks above $\alpha \sim 25^{\circ}$ are further reduced in magnitude compared to F.S. 142 because the primary vortices are lifted further from the surface. The primary vortex footprints can still be observed at $\alpha>25^{\circ}$, Figs. 6(b) and (c), but the footprints are more diminished in magnitude compared to those at F.S. 142. For $\alpha=34.3^{\circ}$ and greater, the peaks in the pressure coefficient curves at $\theta \sim 48^{\circ}$ to $60^{\circ}$ and $\theta \sim 300^{\circ}$ to $312^{\circ}$ are the result of local separation caused by the aircraft production pitot-static probes mentioned previously in the Experiment Description section. Again, at this location, the pressure distributions are nearly symmetrical with only small differences in the primary vortex footprint resulting from discontinuities caused by the refueling probe doors.

The general trend in the data from the forebody is for the maximum suction pressure peaks to first appear at $\alpha \sim 20^{\circ}$ and increase in magnitude as angle of attack is increased. In addition, the footprints of the primary vortex first appear at $\alpha \sim 25^{\circ}$ at F.S. 142 and F.S. 184 and progress forward toward the nose apex as the angle of attack is increased.

In Fig. 7, forebody pressure distributions are presented for angles of attack from $9.9^{\circ}$ to $38.5^{\circ}$ and at Mach 0.60. In this figure, the value for the pressure coefficient corresponding to the local speed of sound $\left(C_{\mathrm{p}}^{*}\right)$ is shown by the asterisk on the abscissa. Because the forebody pressure coefficients were more positive than $C_{p}^{*}$ for all cases presented the flow was subsonic. The data at $M_{\infty}=0.60$ show similar trends to the data previously discussed. Maximum suction pressure pairs at the first three fuselage stations first became evident at $\alpha=19.1^{\circ}$, Fig. 7(a), and increased in magnitude as angle of attack increased to $38.5^{\circ}$, Fig. 7(b). The footprints of the primary vortex were evident at $\alpha=$ $38.5^{\circ}$ at F.S. 85 , and at $\alpha=30.0^{\circ}$ and $38.5^{\circ}$ at F.S. 107 .

At F.S. 142, the effect of the side-mounted ECM antennas can be seen again in the pressure distributions near $\theta=90^{\circ}$ and $270^{\circ}$, Fig. 7. The maximum suction pressure pairs peak at $\theta \sim 68^{\circ}$ and $292^{\circ}$. The footprints of the primary vortex pairs at $\theta \sim 160^{\circ}$ and $200^{\circ}$ first appear at $\alpha=25.6^{\circ}$, Fig. 7(b), and are maximum at the highest angle of attack reached, $38.5^{\circ}$. 
The magnitude of the pressure distributions at F.S. 184, Fig. 7, are generally flatter and at or below the magnitude of those at F.S. 142. The effect of the production pitot-static probes can be seen again at $\alpha=$ $38.5^{\circ}$ and at $\theta=48^{\circ}$ and $312^{\circ}$, Fig. 7(b).

\section{Effect of Mach Number}

Figure 8 shows the data for all five orifice stations on the fuselage for Mach 0.26 to 0.60 for $\alpha \sim 31^{\circ}$. Only a very small effect of Mach number is noted at the fuselage locations for the Mach number range reported. At the first four stations, the $M_{\infty}=0.60$ data are slightly more negative than the data at $M_{\infty}=0.26$. At F.S. 184 the data at $M_{\infty}=0.26$ are slightly more negative.

Also given in Fig. 8 are the effective Reynolds numbers based on local maximum fuselage diameter corrected for angle of attack $\left(R e_{d^{\prime}}\right)^{17}$. Reynolds numbers based on diameter $\left(R e_{d}\right)$ are commonly used to describe the flow about cylinders. Reynolds numbers based on diameter $\left(R e_{d}\right)<2 \times 10^{5}$ are generally considered subcritical where the flow is laminar and laminar separation occurs. At $R e_{d}$ between $2 \times 10^{5}$ and $4 \times 10^{5}$, the flow is critical and laminar separation is followed by turbulent reattachment enclosing a bubble. Reynolds numbers based on diamter $\left(R e_{d}\right)$ greater than $4 \times 10^{5}$ are supercritical or hypercritical where transition moves forward, eventually to the vicinity of the forward stagnation point. In Fig. 8, all data are in the supercritical Reynolds number range or higher for cylinders. At this Reynolds number range, a limited amount of data for cylinders in cross flow in Ref. 17 would predict a significant Mach number effect which was definitely not observed in the flight data shown in Fig. 8.

\section{Correlation of Forebody Pressure Distributions with Flow Visualization Data}

Surface flow visualization using the emitted fluid technique was previously obtained on the F-18 HARV. ${ }^{12-14}$ Selected results are presented in Fig. 9 for angles of attack of $30^{\circ}$ and $47^{\circ}$. Noted in the figures are the primary vortex separation lines $\left(S_{1}\right)$, secondary vortex separation lines $\left(S_{2}\right)$, reattachment lines $(R)$, and laminar separation bubbles (LSBs), as determined by surface flow visualization. Also shown in Fig. 9(a) is a schematic of the flow about the forebody.
In Fig. 10 these results are correlated with forebody flight pressure distributions at $\alpha=30.0^{\circ}$ and $48.1^{\circ}$. The surface flow visualization and the forebody pressure measurements were obtained on separate flights. Some variation in the results can therefore be expected because of slightly different test conditions and test techniques.

At F.S. 70, (Fig. 10(a)) for $\alpha \sim 30^{\circ}$ and at F.S. 70, F.S. 85, and F.S. 107 (Fig. 10(b)) for $\alpha \sim 47^{\circ}$, LSBs were identified in the surface flow visualization. ${ }^{14}$ Laminar separation bubbles are more closely identified with the critical Reynolds number range $\left(2 \times 10^{5}\right.$ $\left.<R e_{d}<4 \times 10^{5}\right)$ than the supercritical range $\left(4 \times 10^{5}\right.$ $\left.<R e_{d}<6 \times 10^{6}\right) .{ }^{17}$ At F.S. 107, Fig. 10(b), the LSBs located by the surface flow visualization at $\theta=113^{\circ}$ and $247^{\circ}$, correlate well with the kinks in the pressure distribution at $\theta=108^{\circ}$ to $114^{\circ}$ and $\theta=246^{\circ}$ to $252^{\circ}$. This is consistent with the discussion in Ref. 20 where the kink or flattening in the pressure distribution was correlated with an LSB for a tangent-ogive cylinder.

Laminar separation bubble kinks in the pressure distribution are also noted for the data at $\alpha=30^{\circ}$ at F.S. 107, Fig. 10(a), though laminar separation bubbles were only noted in the surface flow visualization near F.S. 70. Unfortunately, the orifices at F.S. 70 and F.S. 85 were not dense enough to define the kinks in the pressure distributions as a result of the LSBs. The kinks on the windward side of the maximum suction pressure peaks at F.S. 107 for $\theta=84^{\circ}$ to $90^{\circ}$ and $\theta=$ $270^{\circ}$ to $276^{\circ}$ are not explained at this time. No deviations in the surface streamlines were detected in the flow visualization at these circumferential locations. The discontinuities of the nosecone-fuselage junction precluded any laminar flow on the forebody beyond F.S. 128.5. The peaks in the pressure distributions for F.S. 142 at $\theta \sim 95^{\circ}$ and $265^{\circ}$ (Fig. 10(a)) are caused by the ECM antenna covers as noted earlier.

The primary separation lines $\left(S_{1}\right)$ as identified by the surface flow visualization in Fig. 9, occurred at the end of pressure recovery on the leeward side of the forebody. This can be seen in Fig. 10(a) at F.S. 142 and F.S. 184 and in Fig. 10(b) at F.S. 85 to F.S. 184. This also agrees well with the data of Refs. 19 and 20 for a cone and an ogive. The secondary vortex separation line $\left(S_{2}\right)$ occurs slightly outboard of the footprints of the primary vortex pairs. This occurs at F.S. 142 and F.S. 184 as shown in Fig. 10(a) and at F.S. 107 to F.S. 184 as shown in Fig. 10(b). 


\section{Comparison with Wind-Tunnel Test Results.}

Forebody pressure distribution test results from a 6 percent-scale F-18 wind-tunnel model ${ }^{4}$ are correlated with flight data at $\alpha \sim 30^{\circ}$ at $M_{\infty} \sim 0.30$ and 0.60 and at $\alpha=50^{\circ}, M_{\infty} \sim 0.20$ in Fig. 11. Wind-tunnel data were available at only three forebody fuselage stations, F.S. 107,142 , and 184.

In Fig. 11(a), flight and wind-tunnel data are presented for $\alpha=30.0^{\circ}$ and $M_{\infty} \sim 0.30$. At these conditions the comparisons are good, with the exception being at F.S. 142 where the wind-tunnel data do not indicate the footprints of the primary vortex pair at $\theta \sim 160^{\circ}$ and $200^{\circ}$. At F.S. 107, there appears to be a kink in the wind-tunnel pressure distribution caused by an LSB at $\theta \sim 120^{\circ}$ to $132^{\circ}$, which is slightly more leeward than the flight values. The model and flight Reynolds numbers $\left(R e_{d^{\prime}}\right)$ for all three stations are all in the supercritical range even though the flight values are almost an order of magnitude greater. The ECM antenna-covers were not simulated on the model and hence the peaks near $\theta=90^{\circ}$ and $270^{\circ}$ do not appear in the wind-tunnel data at F.S. 142.

At $\alpha \sim 30^{\circ}$ and $M_{\infty}=0.60$, Fig. 11(b), there were only fair comparisons between flight and wind-tunnel test results. At these conditions, the wind-tunnel maximum suction pressure peaks were approximately $\Delta C_{p}=0.1$ below the flight values. The kinks in the pressure distributions caused by the LSB at F.S. 107 again are present and slightly offset. In addition, the primary vortex footprints at F.S. 142 again were not evident in the wind-tunnel data.

Results from flight and wind-tunnel tests at $\alpha=$ $50.0^{\circ}$ and $M_{\infty} \sim 0.23$ are presented in Fig. 11(c). At these conditions the comparison of the results is mixed. The model Reynolds numbers $\left(R e_{d^{\prime}}\right)$ in this case are critical, whereas the flight values are supercritical. The footprints of the primary vortex are evident for both the flight and wind-tunnel data. At F.S. 107, the windtunnel maximum suction pressure coefficients are approximately 0.2 below the flight values, however, the location of the LSBs and the pressure distributions at the primary vortex footprints agree well. At F.S. 142 the data at the maximum suction pressure peaks compare well but the comparison is not as good at the primary vortex footprints. At F.S. 184, there appears to be an asymmetry in the wind-tunnel pressure distribution that does not appear in the flight data.

\section{Leading-Edge Extension Results}

Examples of the off-surface flow visualization obtained on the F-18 HARV from Ref. 15 are shown in Fig. 12. As can be seen, the flow over the F-18 LEX at high angles of attack is dominated by strong vortical flow. The vortical flow begins at approximately $10^{\circ}$ angle of attack and as the angle of attack increases, the vortex core breakdown point moves forward toward the LEX apex, as shown in the figure.

\section{Effect of Angle of Attack}

The effect of angle of attack on the LEX surface static pressure coefficients is presented in Fig. 13 for angles of attack from $10^{\circ}$ to $50^{\circ}$ at the low speed, 1$g$ flight conditions. Pressure coefficients are plotted from the LEX as a function of LEX span $(y / s)$ as defined previously in Fig. 3. As the aircraft angle of attack increases from $10.0^{\circ}$ to $25.8^{\circ}$, Fig. 13(a) and (b), the LEX maximum suction pressure peaks increase in magnitude and move inboard. Note the change in scale for pressure coefficient for the data from the forebody data. The maximum suction pressures are much greater on the LEX than shown on the forebody. At F.S. 357, $\alpha \sim 30^{\circ}$ and above, (Fig. 13(b) and (c)) the effect of the LEX vortex core breakdown on the pressure distribution can be seen. At $\alpha=30^{\circ}$, vortex core breakdown occurs very near F.S. 357 and moves forward as angle of attack is increased. This causes a marked decrease in the maximum suction pressure peaks and a flattening of the pressure distributions at $\alpha \geq 30^{\circ}$. Similar trends are noted at F.S. 296 and F.S. 253 for $\alpha=39.3^{\circ}$ and $45.4^{\circ}$, respectively. At the highest angles of attack, Fig. 13(c), the flow becomes less symmetrical, particularly at F.S. 253.

The pressure distributions from the leading-edge extensions at $M_{\infty}=0.60$ are presented in Fig. 14. The trends described for the data at $M_{\infty} \sim 0.30$ (Fig. 13) hold for the data at the higher Mach numbers. That is, the inward progression of the maximum suction peaks, the increase in magnitude of the maximum suction pressure peaks up to vortex core breakdown, and then the decrease and general flattening of the pressure distribution beyond the LEX primary vortex core breakdown. Also shown in Figs. 13 and 14 is the value for $C_{p}^{*}$ as marked by the asterisk on the abscissa. Supersonic flow is noted on the LEX for angles of attack of approximately $19^{\circ}$ and greater. 


\section{Effect of Mach Number}

In Fig. 15 the effects of Mach number on the pressure distributions on the LEXs are summarized for $\alpha \sim$ $31^{\circ}$. At all three LEX orifice stations, a significant effect of compressibility is shown by a reduction in vortex-induced suction pressure as Mach number is increased. Even the data at F.S. 357, where the suction pressures are influenced by the vortex core breakdown, were affected.

\section{Correlation of Leading-Edge Extension Pressure} Distributions with Flow Visualization

Selected surface flow visualization results obtained previously on the F-18 HARV and reported in Refs. 12 and 13 are presented in Fig. 16 for $\alpha \sim 30^{\circ}$ and $47^{\circ}$. Noted in the photos are the secondary $\left(S_{2}\right)$ and tertiary separation lines $\left(S_{3}\right)$ as defined by the merging of the surface streamlines. Also shown in the figure is a schematic of the flow about the LEX.

The data from the surface flow visualization have been correlated with the pressure distributions obtained on the LEX in Fig. 17 at $\alpha=30.0^{\circ}$ and $48.1^{\circ}$. Surface flow visualization was obtained only on the left LEX. However, the separation line locations are shown on both sides for comparison with the pressure distributions since they were obtained at approximately $0^{\circ}$ sideslip and symmetry is assumed. At $\alpha \sim$ $30^{\circ}$, Fig. $17(\mathrm{a})$, the secondary separation lines $\left(S_{2}\right)$ correspond well with the end of pressure recovery outboard of the maximum suction pressure peaks. The tertiary separation lines $\left(S_{3}\right)$ seem to correspond with the end of pressure recovery inboard of the secondary suction peak near the LEX leading edge. The lateral location of the primary vortex cores on the F-18 HARV were determined with smoke visualization. ${ }^{15}$ Their locations are shown in Fig. 17(a) at F.S. 296 and F.S. 357 and they were located just inboard of the maximum suction pressure peaks. This agrees well with the latcral position of vortex cores on a sharp delta wing suggested by Hummel and Redeker ${ }^{21}$ and corroborated by Seshadri and Bütefisch ${ }^{22}$-which showed the lateral position of the vortex core coincided closely with the maximum suction peak.

At $\alpha=48.1^{\circ}$, Fig. $17(\mathrm{~b})$, the LEX primary vortex core breakdown occurred forward of F.S. 253 and all three stations experienced turbulent, buffeting-vortical flow while the aircraft was in a mild wing rock. At F.S. 296 and F.S. 357 the pressure distributions were essentially flat and did not have distinct suction pres- sure peaks. At F.S. 253, the pressure distribution was slightly asymmetrical and the end of pressure recovery did not correspond as well with the separation lines. This was partly because of the unsteadiness of the flow and the difficulty in locating the separation lines at this condition.

\section{Correlation of Leading-Edge Extension Pressure Distributions with Wind-Tunnel Test Results}

In Fig. 18 the LEX pressure distribution test results obtained from a 6-percent-scale F-18 wind-tunnel model, ${ }^{4}$ are correlated with flight data at $\alpha \sim 30^{\circ}$, $M_{\infty} \sim 0.30$ and 0.60 and at $\alpha=50^{\circ}, M_{\infty} \sim 0.20$. Wind-tunnel data were available from only the upper surface of the LEX. At $\alpha \sim 30^{\circ}$, Fig. 18(a) and (b), the wind turnel tends to underpredict slightly the suction pressures at F.S. 253 and F.S. 296. The correlation was good at F.S. 357. Both the flight and wind-tunnel test results indicate some asymmetry in the LEX pressure distributions at $\alpha=50^{\circ}$, Fig. 18(c).

\section{Concluding Remarks}

Pressure distributions have been reported at angles of attack from $10^{\circ}$ to $50^{\circ}$ and at Mach 0.23 to 0.60 at five fuselage stations on the forebody and at three fuselage stations on the leading-edge extensions (LEXs) of the NASA F-18 high alpha research vehicle (HARV). The reported results have been correlated with flow visualization results obtained during a previous investigation on the F-18 HARV and with results obtained from a 6-percent-scale F-18 wind-tunnel model.

The general trend in the data from the forebody was for the maximum suction pressure peaks to first appear at angle of attack $(\alpha) \sim 19^{\circ}$ and increase in magnitude as angle of attack was increased. In addition, the "footprint" of the primary vortex pairs first appeared at $\alpha \sim 25^{\circ}$ at F.S. 142 and F.S. 184 and progressed forward toward the nose apex as the angle of attack was increased.

The general trend of the LEX pressure distribution was the inward progression of the maximum suction peaks, the increase in magnitude of the maximum suction pressure peaks up to vortex core breakdown, and then the decrease and general flattening of the pressure distribution beyond the LEX primary vortex core breakdown.

No significant effect of Mach number was noted for the forebody results for the Mach number range reported. However, at all three LEX orifice stations a 
substantial compressibility cffect resulted in a significant reduction in vortex-induced suction pressure as Mach number increased.

The forebody primary separation line $\left(S_{1}\right)$ as identified by the surface flow visualization, correlated well with the end of pressure recovery lecward of the maximum suction pressure peaks. The location of the laminar separation bubble (LSB) correlated well with the kinks in the pressure distribution curves windward of the maximum suction peaks.

The LEX secondary vortex separation lines, as determined from flow visualization, corresponded well with the end of pressure recovery outboard of the maximum suction pressure peak. The location of the LEX primary vortex core, as determined by using smoke visualization, was just inboard of the LEX maximum suction peak.

Comparisons with forebody pressure distribution test results from a 6-percent scale F-18 windtunnel model were good at free-stream Mach number $\left(M_{\infty}\right) \sim 0.30$ and $\alpha=30^{\circ}$, with the exception of the lack of footprints of the primary vortex pairs in the wind-tunnel data. At $\alpha=50^{\circ}$, the windtunnel test results showed the presence of the footprints but also exhibited some asymmetry not present in the flight results.

On the LEX, at $\alpha \sim 30^{\circ}$, the wind tunnel tends to underpredict slightly the suction pressures at some locations. Some asymmetry in the LEX pressure distributions at $\alpha=50^{\circ}$ was observed for both the flight and wind-tunnel test results.

\section{References}

${ }^{1}$ Skow, A.M., and Erickson, G.E., Modern Fighter Aircraft Design for High-Angle-of-Attack Maneuvering, AGARD LS-121, paper no. 4, 1982.

${ }^{2}$ Erickson, Gary E., Water Tunnel Flow Visualization and Wind Tunnel Data Analysis of the F/A-18, NASA CR-165859, 1982.

${ }^{3}$ Banks, Daniel W., "Wind-Tunnel Investigation of the Forebody Aerodynamics of a Vortex-Lift Fighter Configuration at High Angles of Attack," SAE Technical Paper Series 881419, Oct. 1988.

${ }^{4}$ Erickson, G.E., Hall, R.M., Banks, D.W., Del Frate, J.H., Schreiner, J.A., Hanlcy, R.J., and Pulley, C.T., "Experimental Investigation of the F/A-18 Vortex Flow at Subsonic Through Transonic Speeds, Invited Papcr," AIAA 89-2222, July-Aug. 1989.
${ }^{5}$ Thomas, James L., Walters, Rober W., Reu, Taekyu, Ghaffari, Farhad, Weston, Robert P., and Luckring, James M., "A Patched-Grid Algorithm for Complex Configurations Directed Towards the F/A-18 Aircraft," AIAA 89-0121, Jan. 1989.

${ }^{6}$ Ghaffari, F., Luckring, J., Thomas, J.L., and Bates, B.L., "Navier-Stokes Solutions about the F/A-18 Forcbody-LEX Configuration," AIAA 890338, Jan. 1989.

${ }^{7}$ Schiff, Lewis B., Cummings, Russell M., Sorenson, Reese L., and Rizk, Yehia M., "Numerical Simulation of High-Incidence Flow over the F-18 Fuselage Forebody," AIAA 89-0339, Jan. 1989.

${ }^{8}$ Schiff, Lewis B., Cummings, Russell M., Sorenson, Rcese L., and Rizk, Yehia M., "Numerical Simulation of F-18 Fuselage Forebody Flow at High Angles of Attack," NASA CP-10038, vol. 1, 1989 , pp. 345-359.

${ }^{9}$ Cummings, Russell M., Rizk, Yehia M., Schiff, Lewis B., and Chaderjian, Neal M., "Navier-Stokes Predictions of the Flowfield Around the F-18 (HARV) Wing and Fuselage at Large Incidence," AIAA 90-0099, Jan. 1990.

${ }^{10}$ Fisher, David F., Richwine, David M., and Banks, Daniel W., Surface Flow Visualization of Separated Flows on the Forebody of an F-18 Aircraft and WindTunnel Model, NASA TM-100436, 1988. Also published as AIAA 88-2112.

${ }^{11}$ Curry, Robert E., and Richwine, David M., "An Airbome System for Vortex Flow Visualization on the F-18 High-Alpha Research Vehicle," AIAA 88-4671-CP, Sept. 1988.

${ }^{12}$ Fisher, David F., and Meyer, Robert R., Jr., Flow Visualization Techniques for Flight Research, NASA TM-100455, 1988. Also published in Flight Test Techniques, AGARD CP 452, paper no. 20, 1988.

${ }^{13}$ Fisher, David F., Del Frate, John H., and Richwine, David M., "In-Flight Flow Visualization Characteristics of the NASA F-18 High Alpha Research Vehicle at High Angles of Attack," SAE Technical Paper Scrics 892222, Sept. 1989.

${ }^{14}$ Schneider, Edward T., and Meyer, Robert R., Jr., "F-18 High Alpha Research Vehicle: Description, Results, and Plans," SETP 33rd Symposium Proceedings, Scpt. 1989.

${ }^{15}$ Del Frate, John H., and Zuniga, Fanny A., "InFlight Flow Field Analysis on the NASA F-18 High 
Alpha Research Vehicle With Comparisons to Ground Facility Data," AIAA-90-0231, 1990.

${ }^{16}$ Whitmore, Stephen A., Moes, Timothy R., and Larson, Terry J., Preliminary Results From a Subsonic High Angle-of-Attack Flush Airdata Sensing (HI-FADS) System: Design, Calibration, and Flight Test Evaluation, NASA TM-101713, 1990.

${ }^{17}$ Polhamus, Edward C., A Review of Some Reynolds Number Effects Related to Bodies at High Angles of Attack, NASA CR-3809, 1984.

${ }^{18}$ Richardson, Noman R., and Pearson, Albin O., Wind-Tunnel Calibrations of a Combined PitotStatic Tube, Vane-Type Flow-Direction Transmitter, and Stagnation-Temperature Element at Mach Numbers From 0.60 to 2.87, NASA TN D-122, 1959.
${ }^{19}$ Pcake, D.J., Fisher, D.F., and McRac, D.S., "Flight, Wind Tunnel, and Numerical Experiments with a Slender Cone at Incidence," AIAA Journal, vol. 20 , no. 10 , Oct. 1982 , p. 1338.

${ }^{20}$ Hall, Robert M., "Influence of Reynolds Number on Forcbody Side Forces for 3.5-Diameter TangentOgive Bodies," AIAA-87-2274, Aug. 1987.

${ }^{21}$ Hummel, D., and Redeker, G., Experimental Determination of Bound Vortex Lines and Flow in the Vicinity of the Trailing Edge of a Slender Delta Wing, NASA Technical Translation, NASA TT F-15, $012,1973$.

${ }^{22}$ Seshadri, S.N., and Bütefisch, Karl-Aloys, "Evaluation of LDA 3-Component Velocity Data on a $65^{\circ}$ Delta Wing at $\mathrm{M}=0.85$ and First Results of an Analysis," DFVLR-FB 89-19, Mar. 1989.

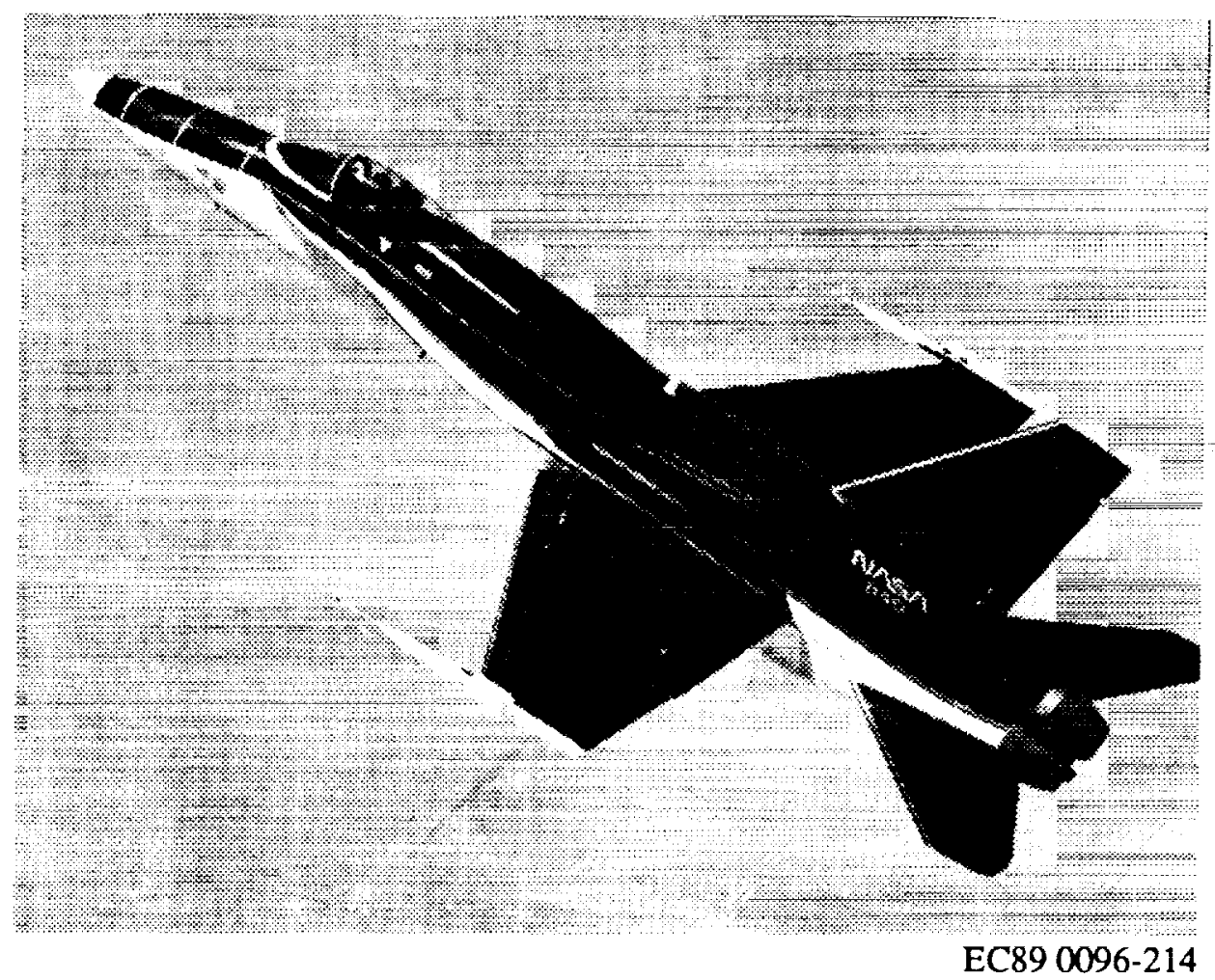

Fig. 1 F-18 HARV. 


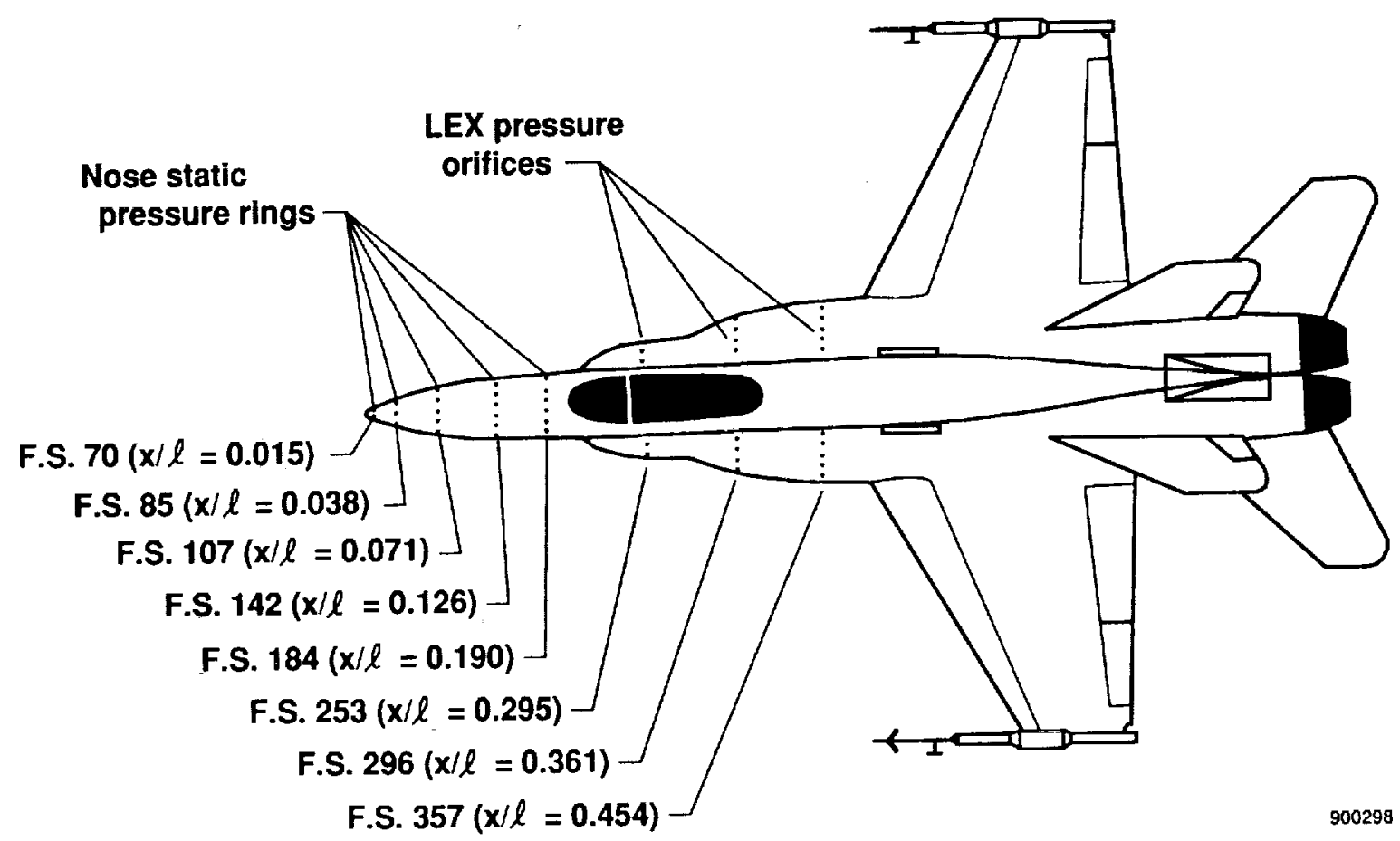

Fig. 2 Forcbody and LEX pressure measurement stations. 

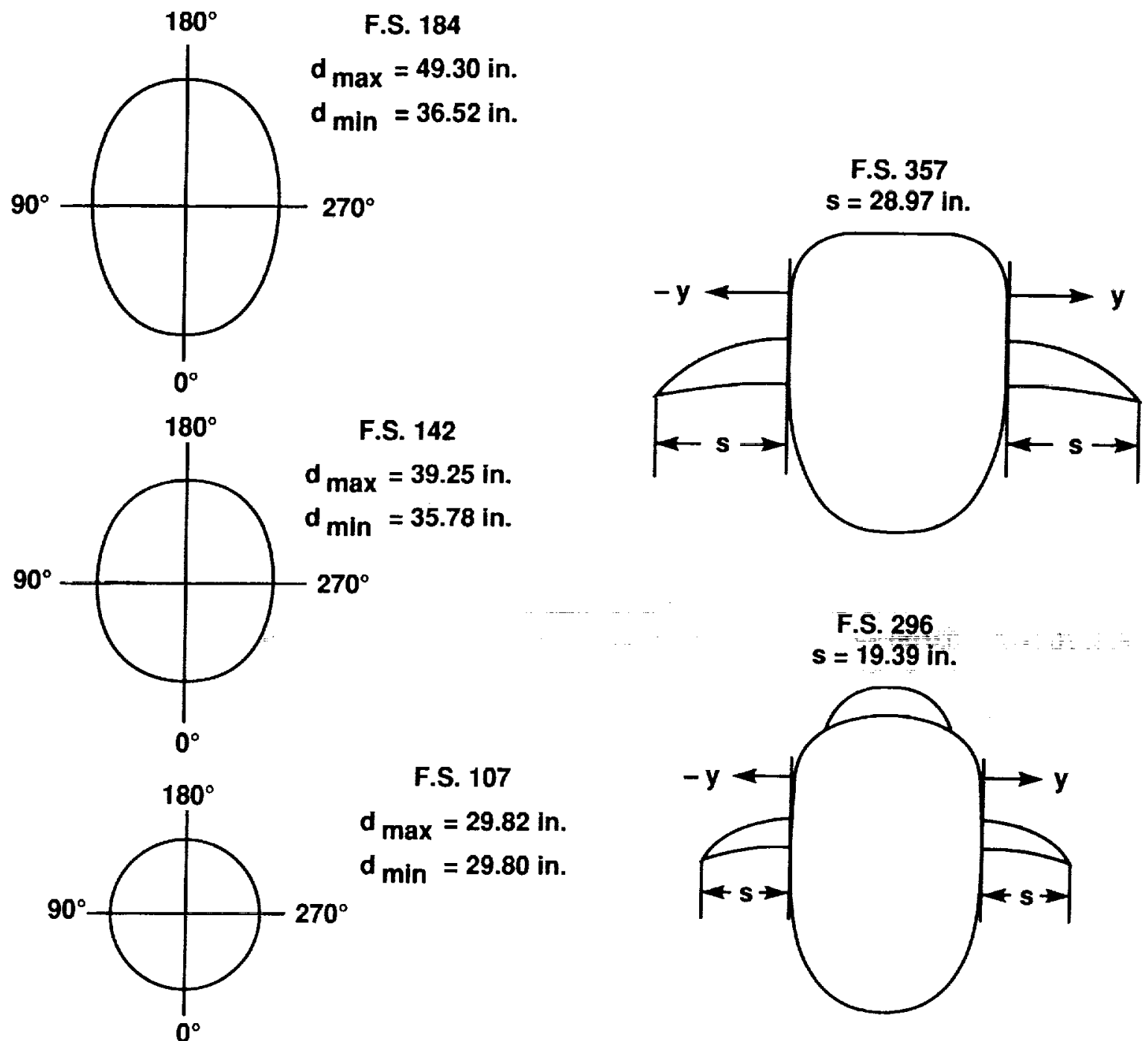

F.S. 107

$d_{\text {max }}=29.82$ in.

$d_{\text {min }}=29.80$ in.

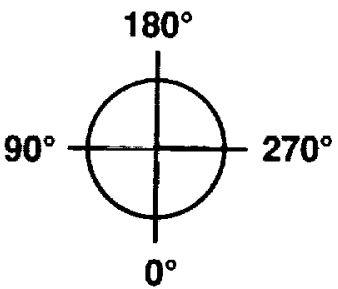

F.S. 85

$d=20.42$ in.
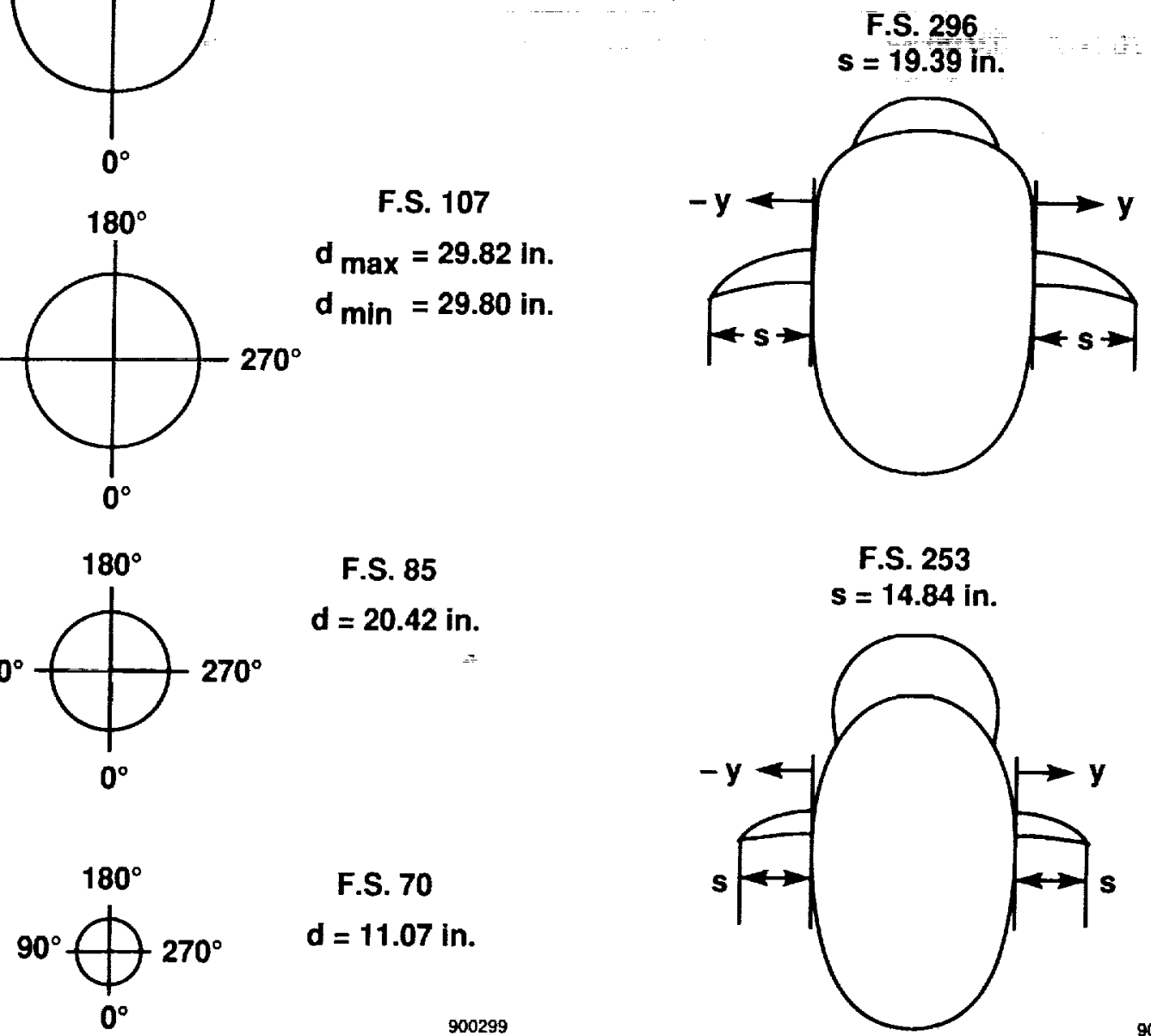

F.S. 70

$d=11.07$ in.

(a) Forcbody circumferential angle.

900299

Fig. 3 Cross sections of pressure measurement stations and orifice orientation, looking aft. 
ORIGINAL PAGE

BLACK AND WHITE PHOTOGRAPH

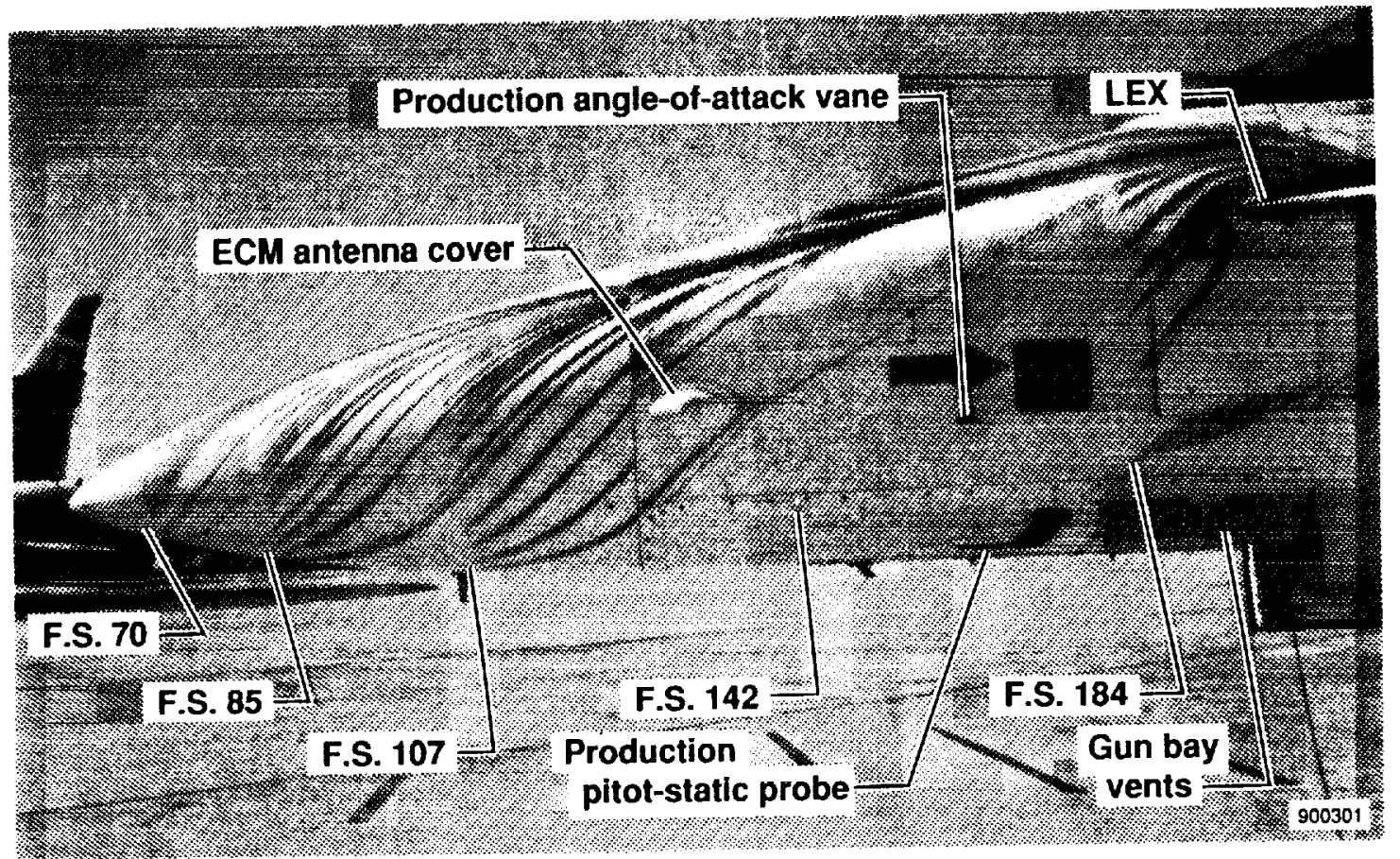

(a) Forcbody locations.

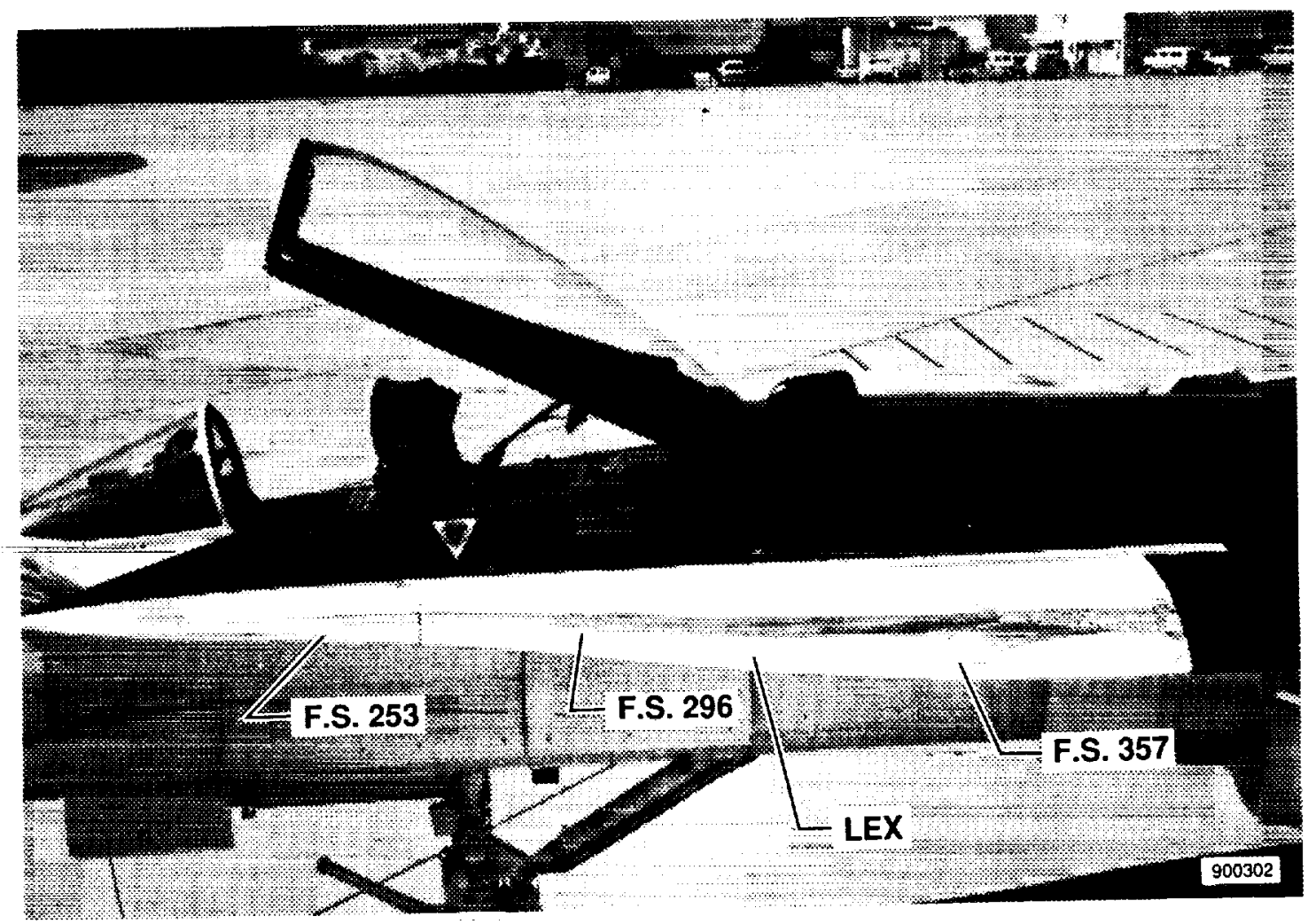

(b) LEX locations.

Fig. 4 Locations of protusions near pressure measurement stations. 


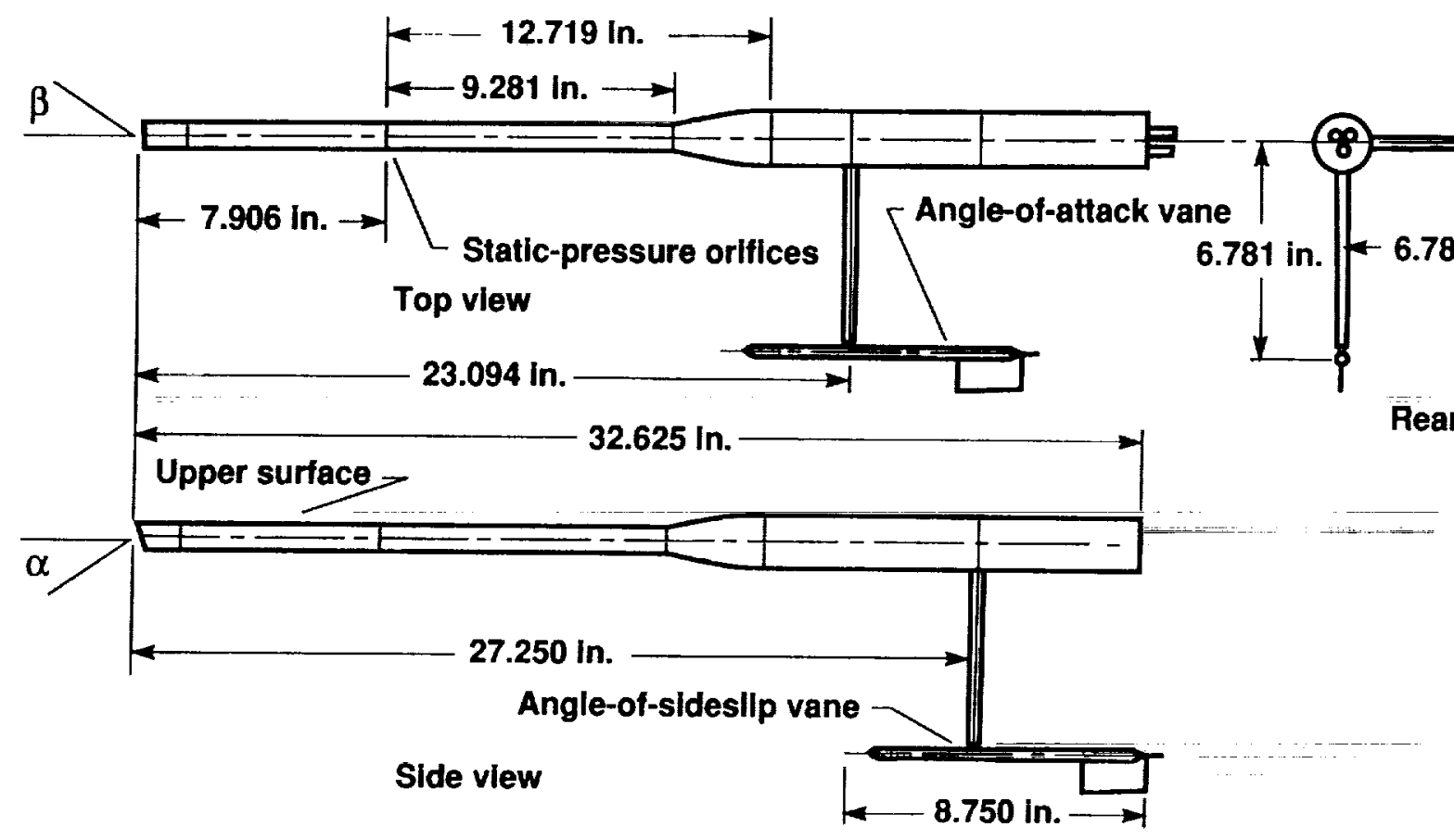

(a) Drawing of NACA airdata probc, right wingtip.

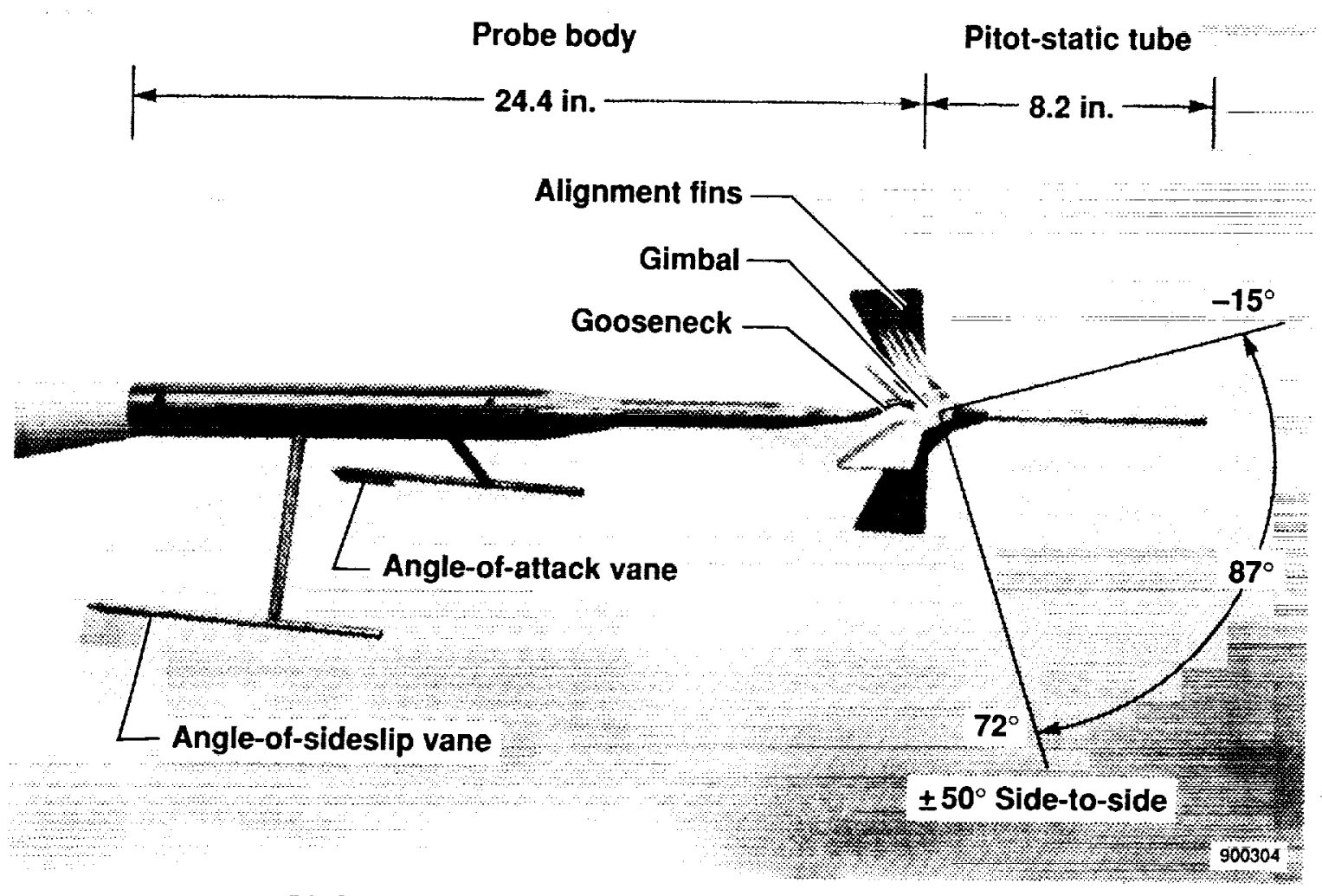

(b) Photo of swiveling-head airdata probe, left wingtip.

Fig. 5 Airdata probes mounted on F-18 HARV wingtips. 

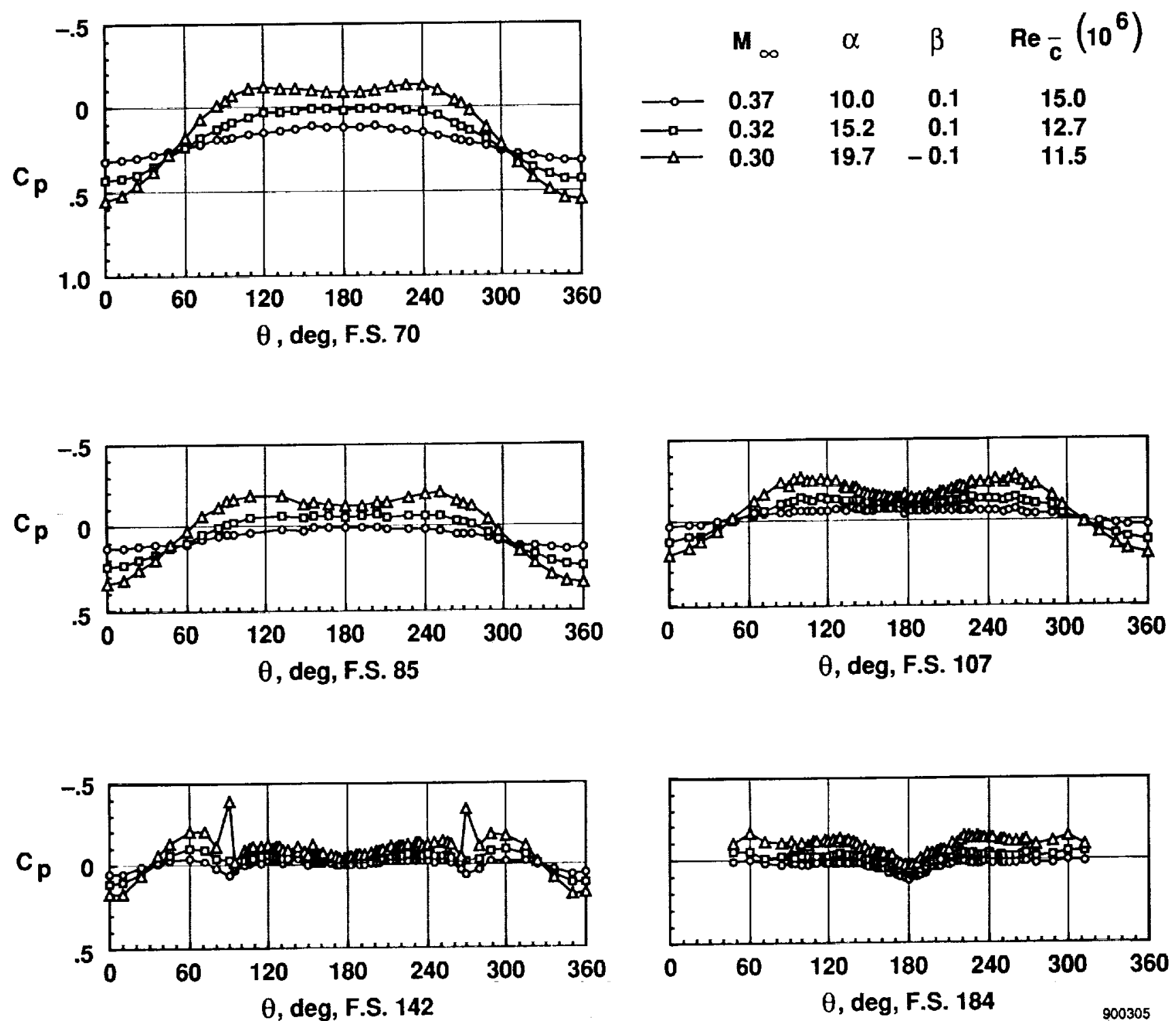

(a) $\alpha=10.0^{\circ}, 15.2^{\circ}$, and $19.7^{\circ} ; M_{\infty} \sim 0.35$.

Fig. 6 Effect of angle of attack on forcbody surface static pressurc cocfficients on the F-18 HARV at low specd. 

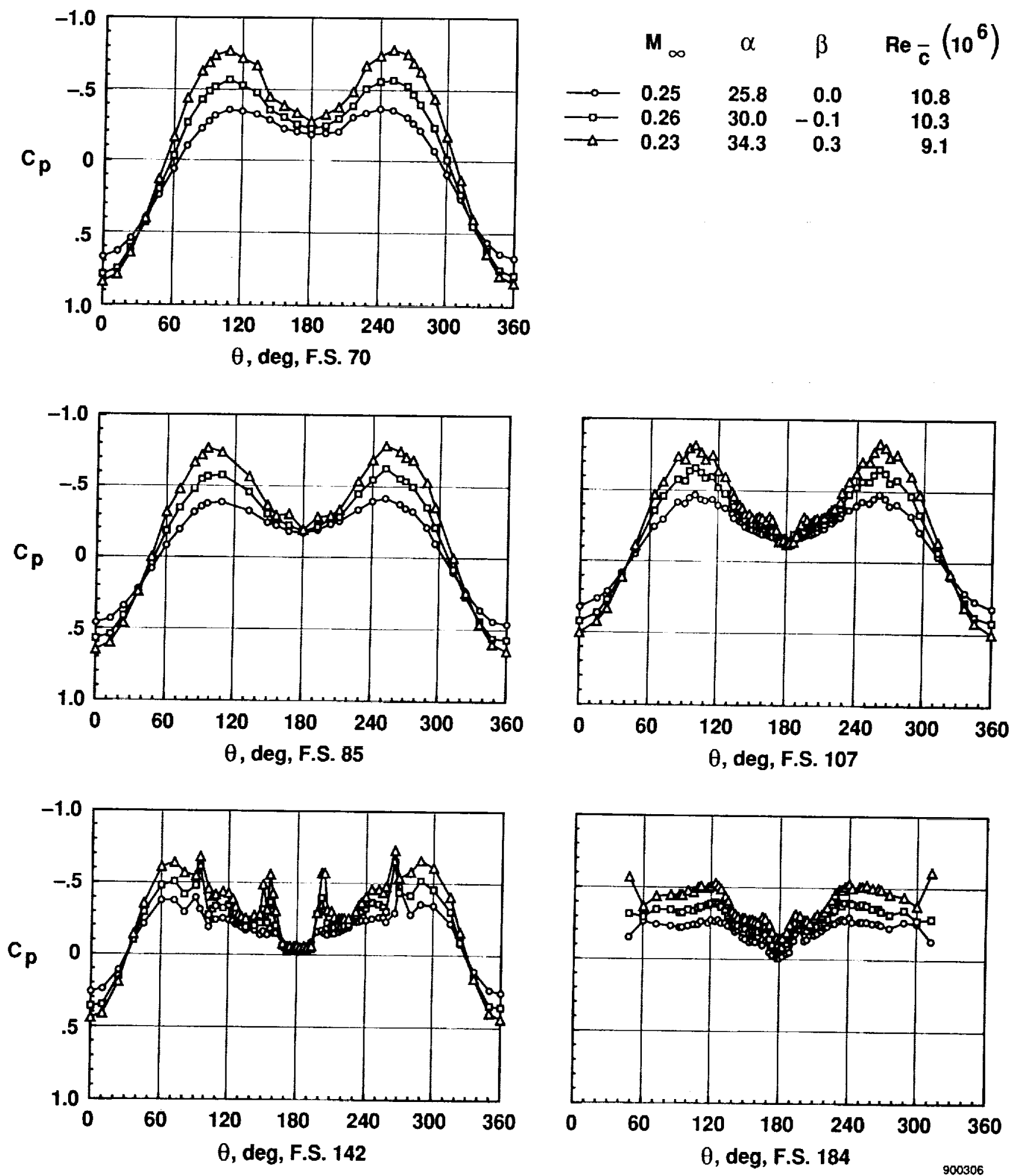

(b) $\alpha=25.8^{\circ}, 30.0^{\circ}$, and $34.3^{\circ} ; M_{\infty} \sim 0.25$.

Fig. 6 Continued. 

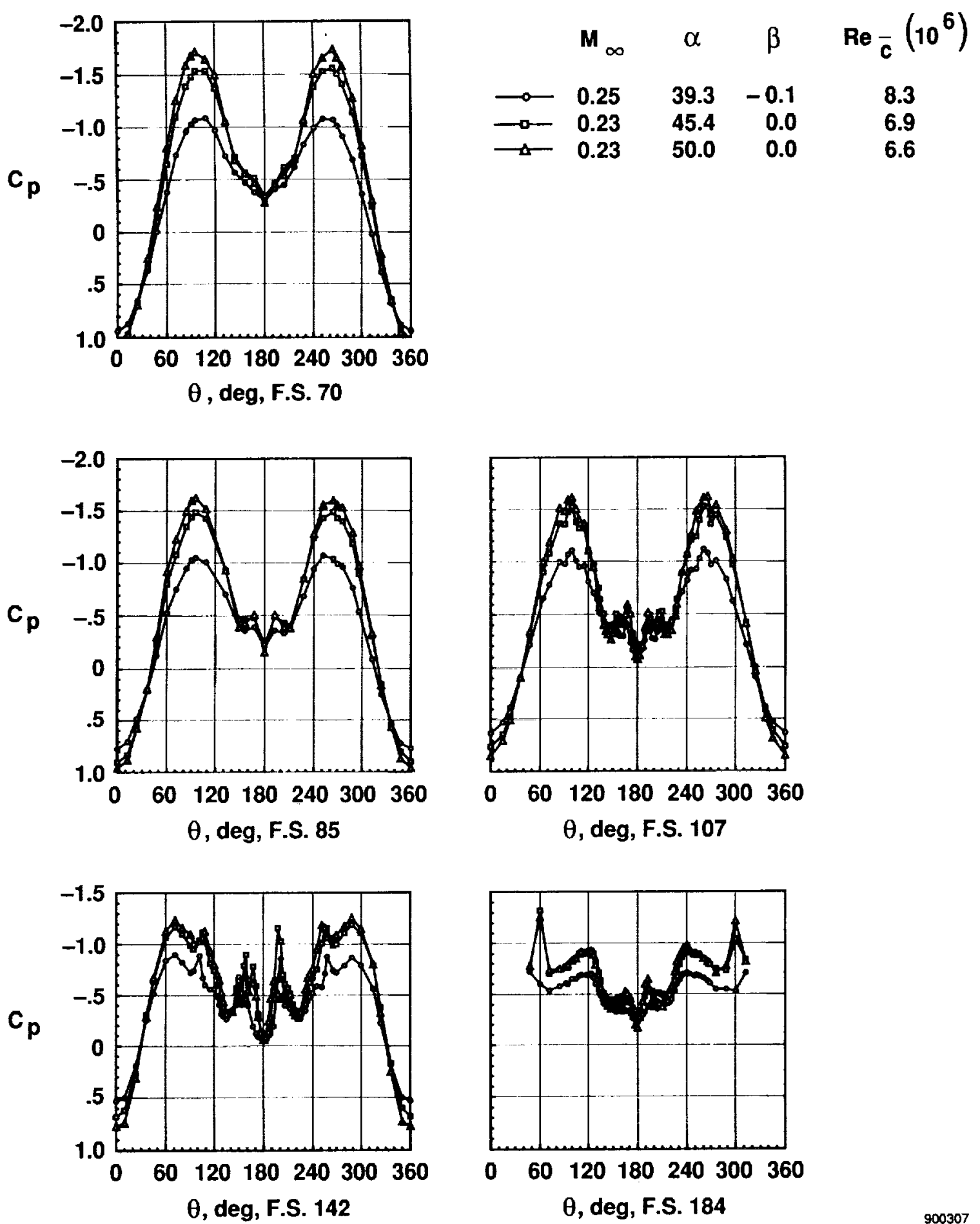

(c) $\alpha=39.3^{\circ}, 45.4^{\circ}$, and $50.0^{\circ} ; M_{\infty} \sim 0.25$.

Fig. 6 Concluded. 

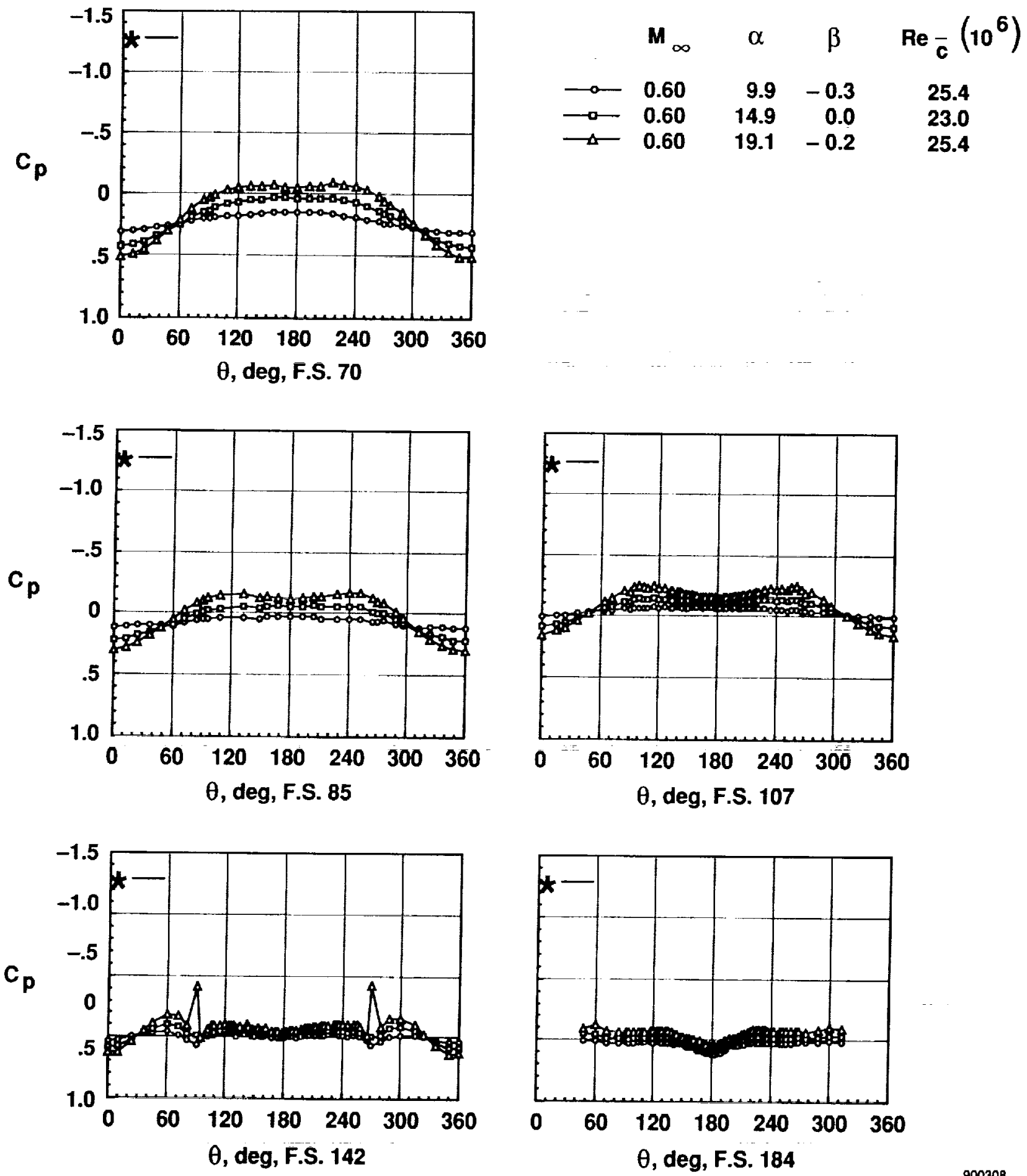

(a) $\alpha=9.9^{\circ}, 14.9^{\circ}$, and $19.1^{\circ}$.

900308

Fig. 7 Effect of angle of attack on forebody surface static pressurc coefficients on the F-18 HARV at $M_{\infty} \sim 0.60$. 


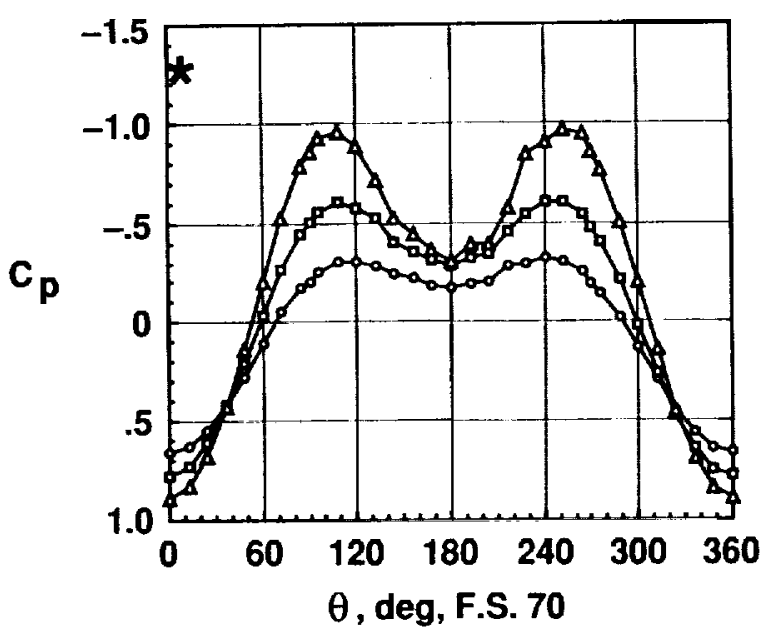

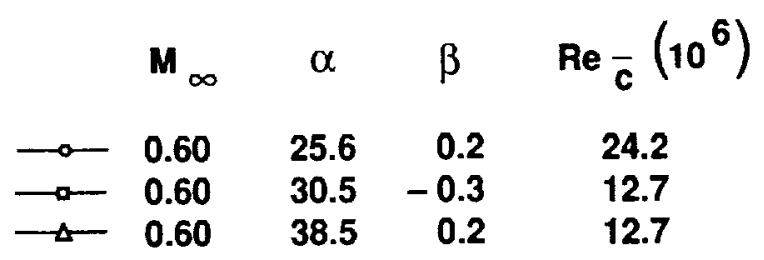
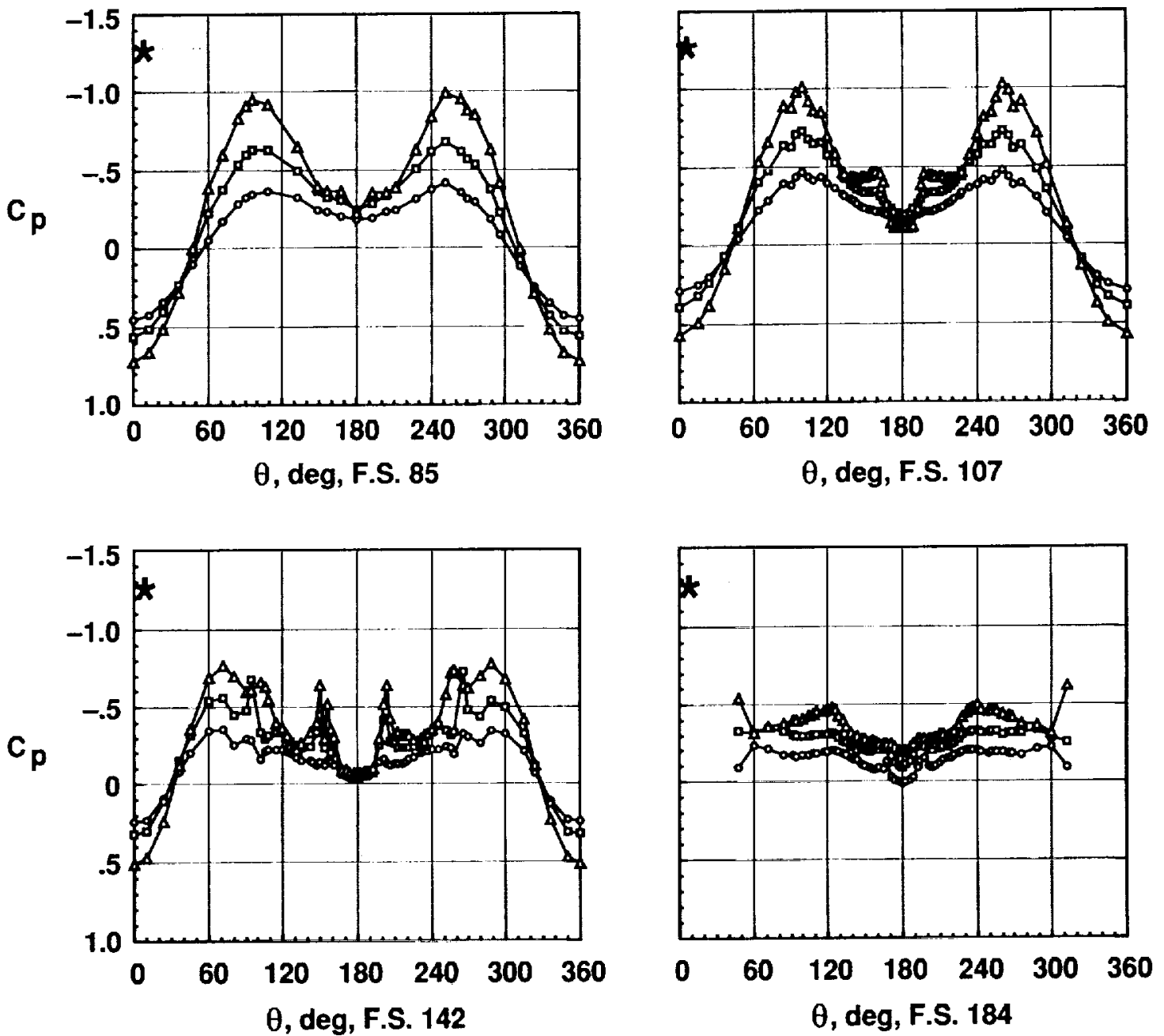

900309

(b) $\alpha=25.5^{\circ}, 30.5^{\circ}$, and $38.5^{\circ}$.

Fig. 7 Concluded. 

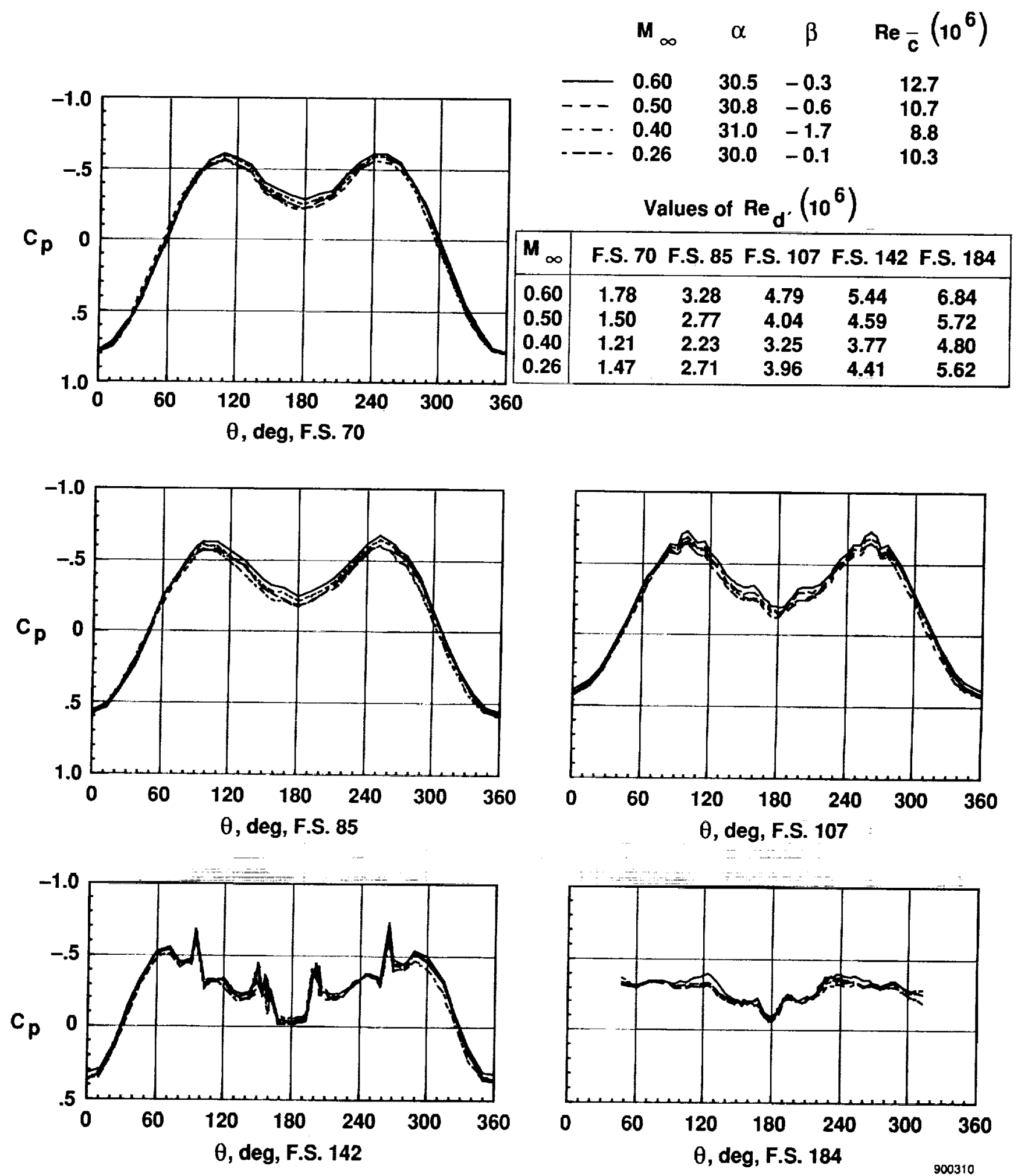

Fig. 8 Effect of Mach number on forcbody surface static pressure coefficients on the F-18 HARV, $\alpha \sim 31^{\circ}$. 


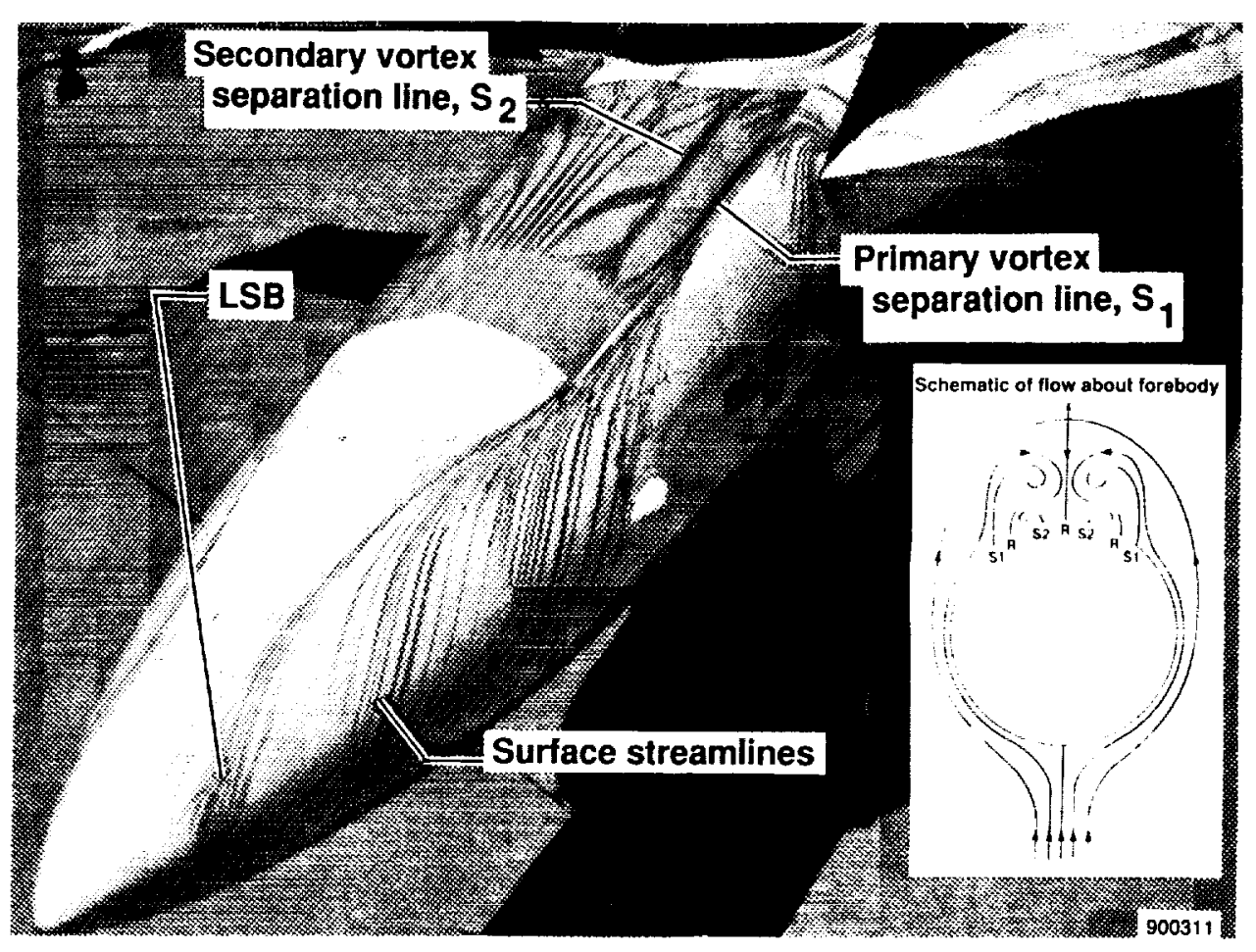

(a) $\alpha \sim 30^{\circ}$.

Fig. 9 Surface flow visualization on F-18 HARV forcbody.

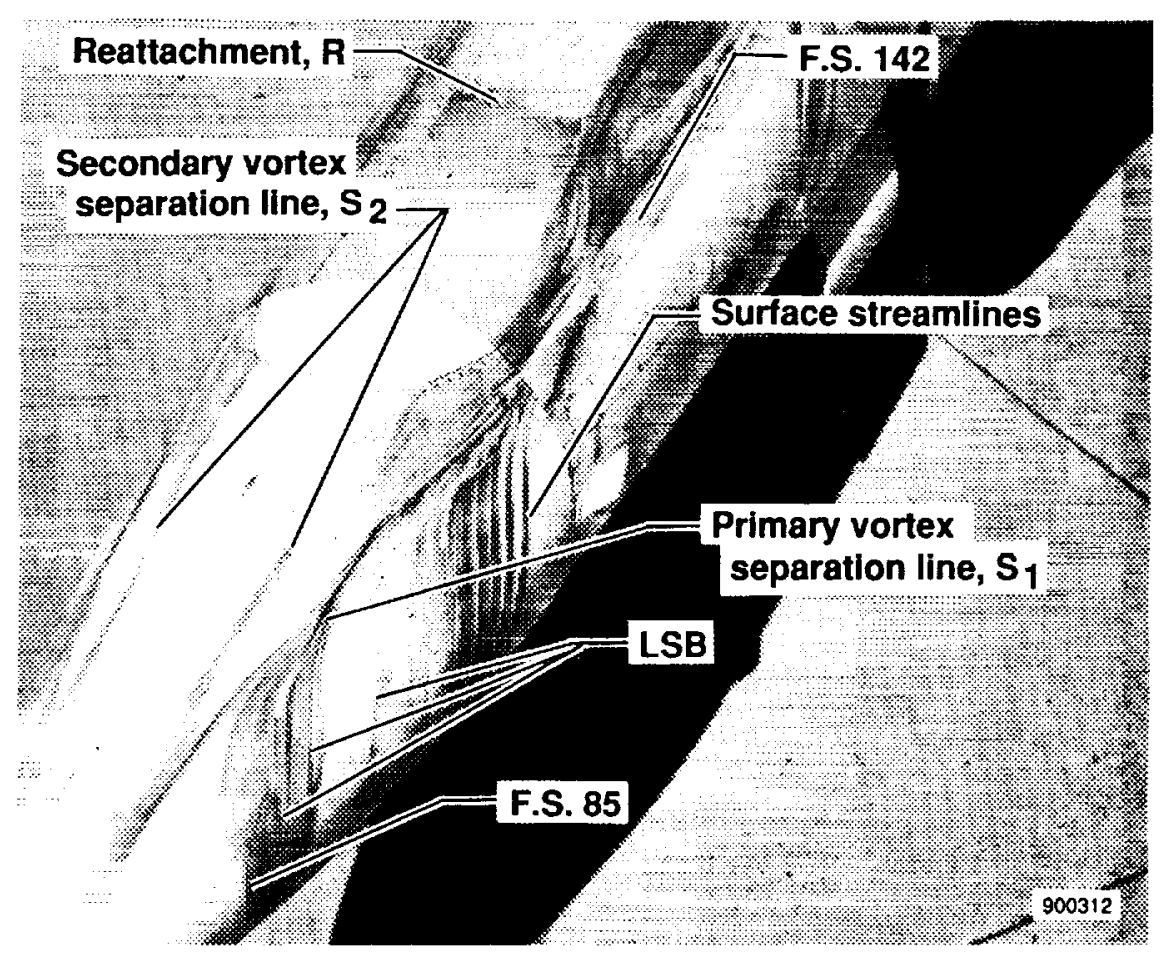

(b) $1 / 4$ vicw, $\alpha \sim 47^{\circ}$.

Fig. 9 Continued. 


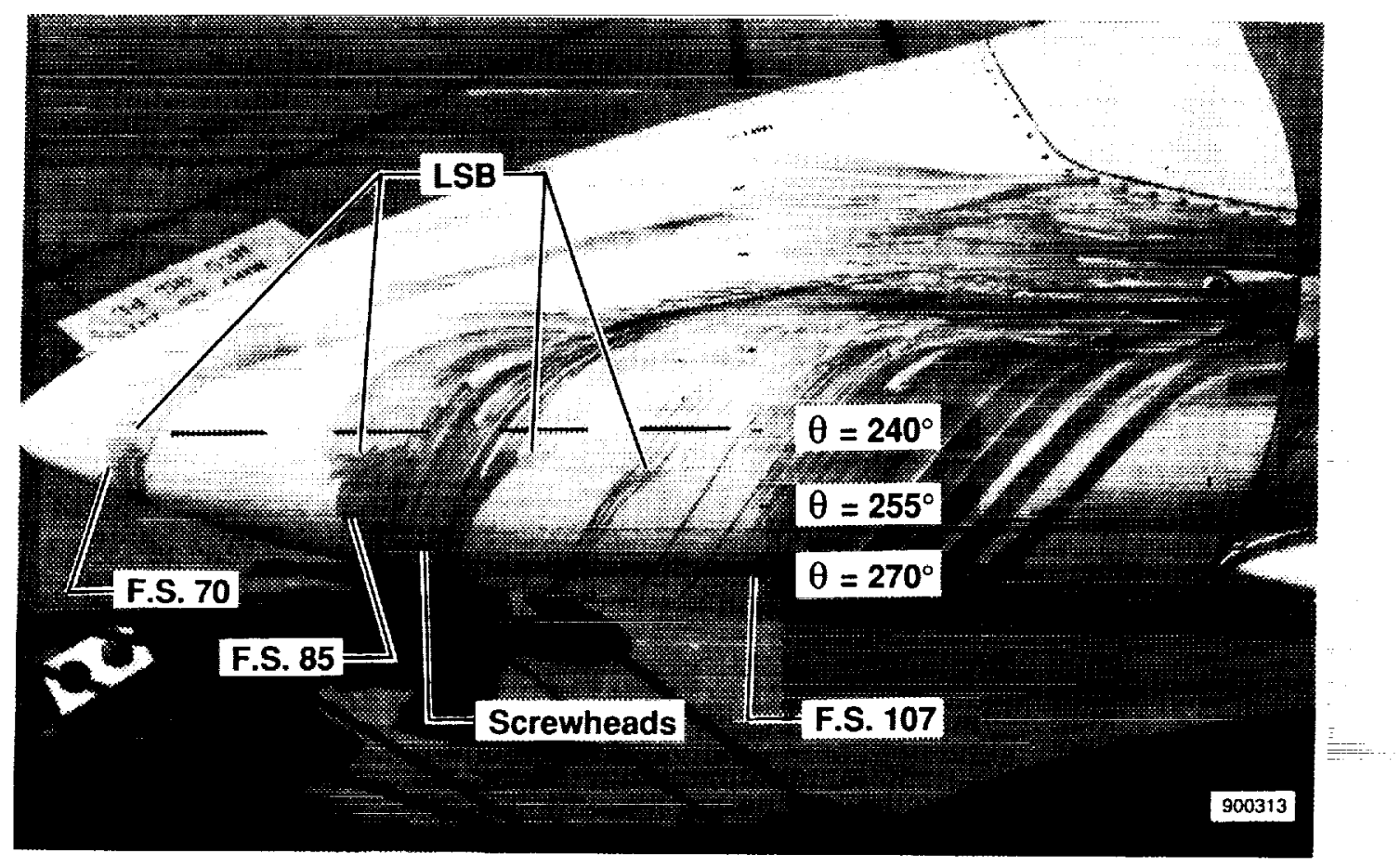

(c) Closcup of nosecone, $\alpha \sim 47^{\circ}$.

Fig. 9 Concluded. 


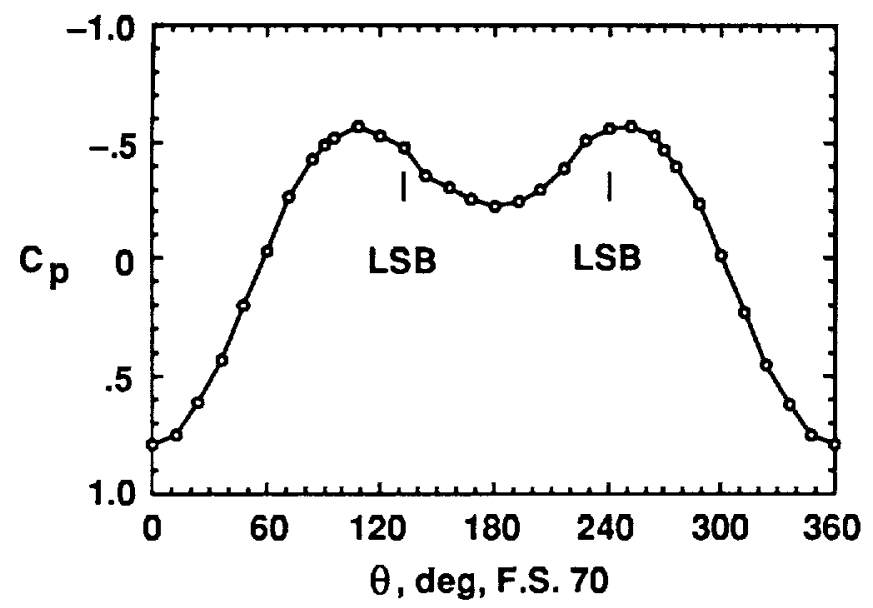

\begin{tabular}{|c|c|c|c|c|}
\hline$\rightarrow c$ & $\begin{array}{l}M_{\infty} \\
.26\end{array}$ & $\begin{array}{rr}\alpha & \beta \\
30.0 & -0 .\end{array}$ & \multicolumn{2}{|c|}{$\begin{array}{c}\operatorname{Re} \frac{c}{c}\left(10^{6}\right) \\
10.3\end{array}$} \\
\hline \multicolumn{5}{|c|}{ Values of $\operatorname{Re}_{\mathrm{d}^{\prime}}\left(10^{6}\right)$} \\
\hline F.S. 70 & F.S. 85 & F.S. 107 & F.S. 142 & F.S. 184 \\
\hline 1.47 & 2.70 & 3.94 & 4.47 & 5.62 \\
\hline
\end{tabular}
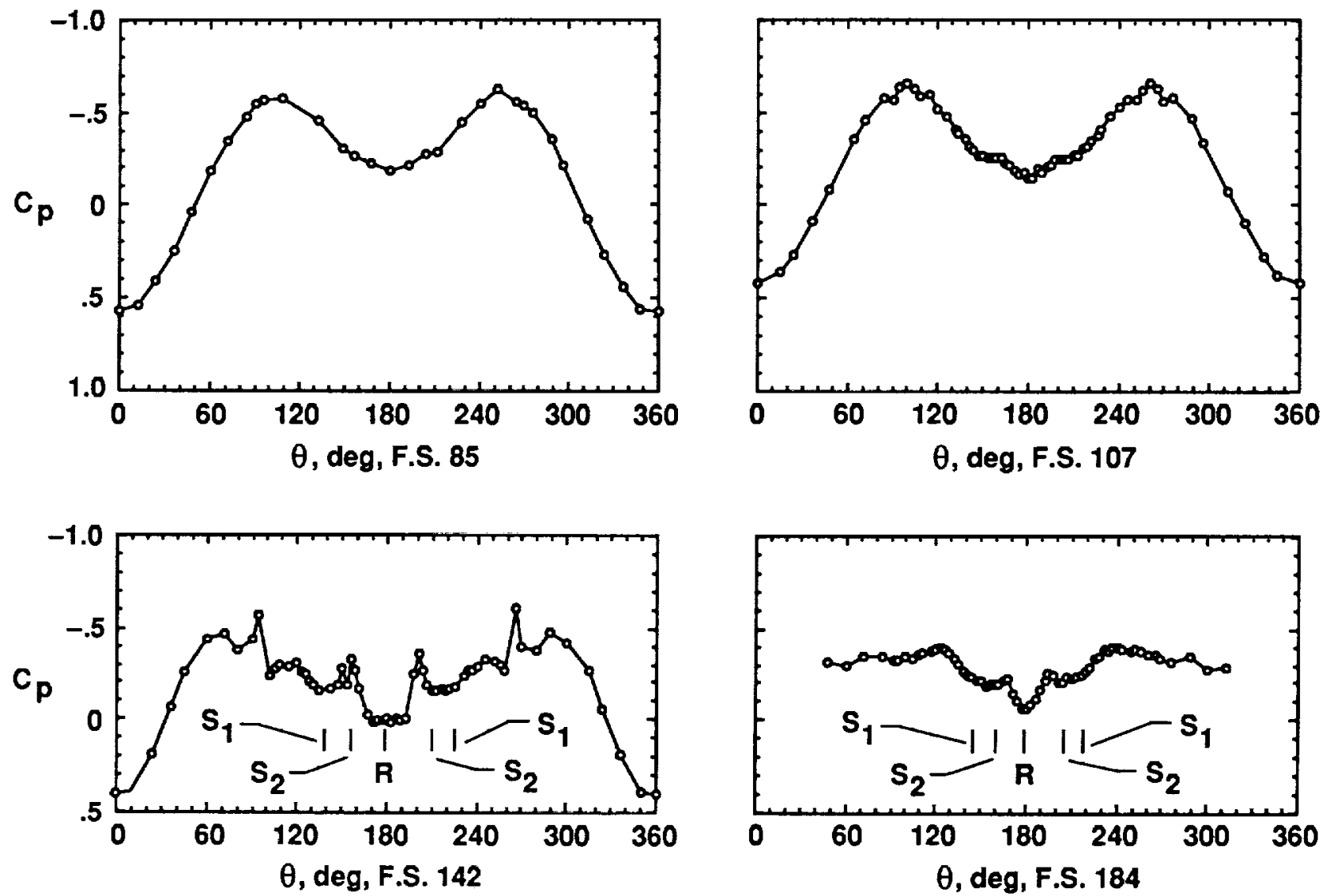

(a) $\alpha=30.0^{\circ} ; M_{\infty}=0.26$.

Fig. 10 Comparison of forebody surface static pressure coefficients with flow visualization results on the F-18 HARV. 


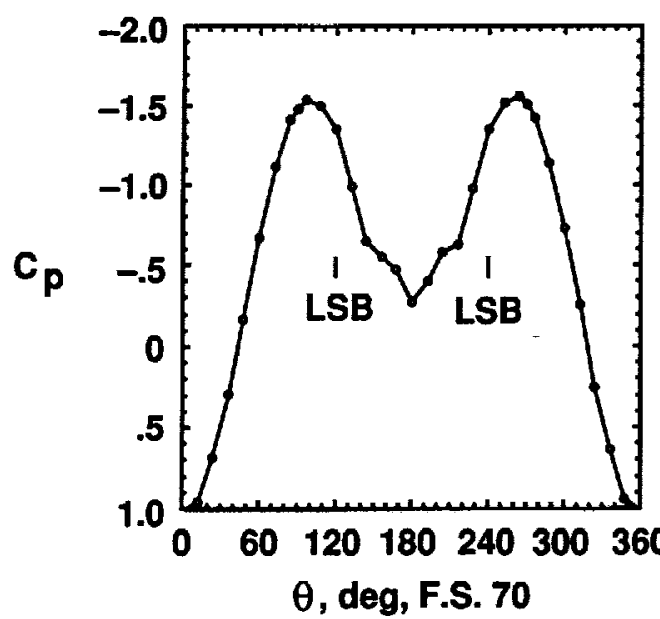

\begin{tabular}{|c|c|c|c|c|}
\hline & $v_{\infty}$ & $\begin{array}{c}\alpha \\
48.1\end{array}$ & $\mathbf{R e}$ & $\begin{array}{l}c\left(10^{6}\right) \\
6.9\end{array}$ \\
\hline \multicolumn{5}{|c|}{ Values of $\mathrm{Re}_{\mathrm{d}^{\prime}}\left(10^{6}\right)$} \\
\hline F.S. 70 & F.S. 85 & F.S. 107 & F.S. 142 & F.S. 184 \\
\hline 0.68 & 1.26 & 1.84 & 2.29 & 2.87 \\
\hline
\end{tabular}
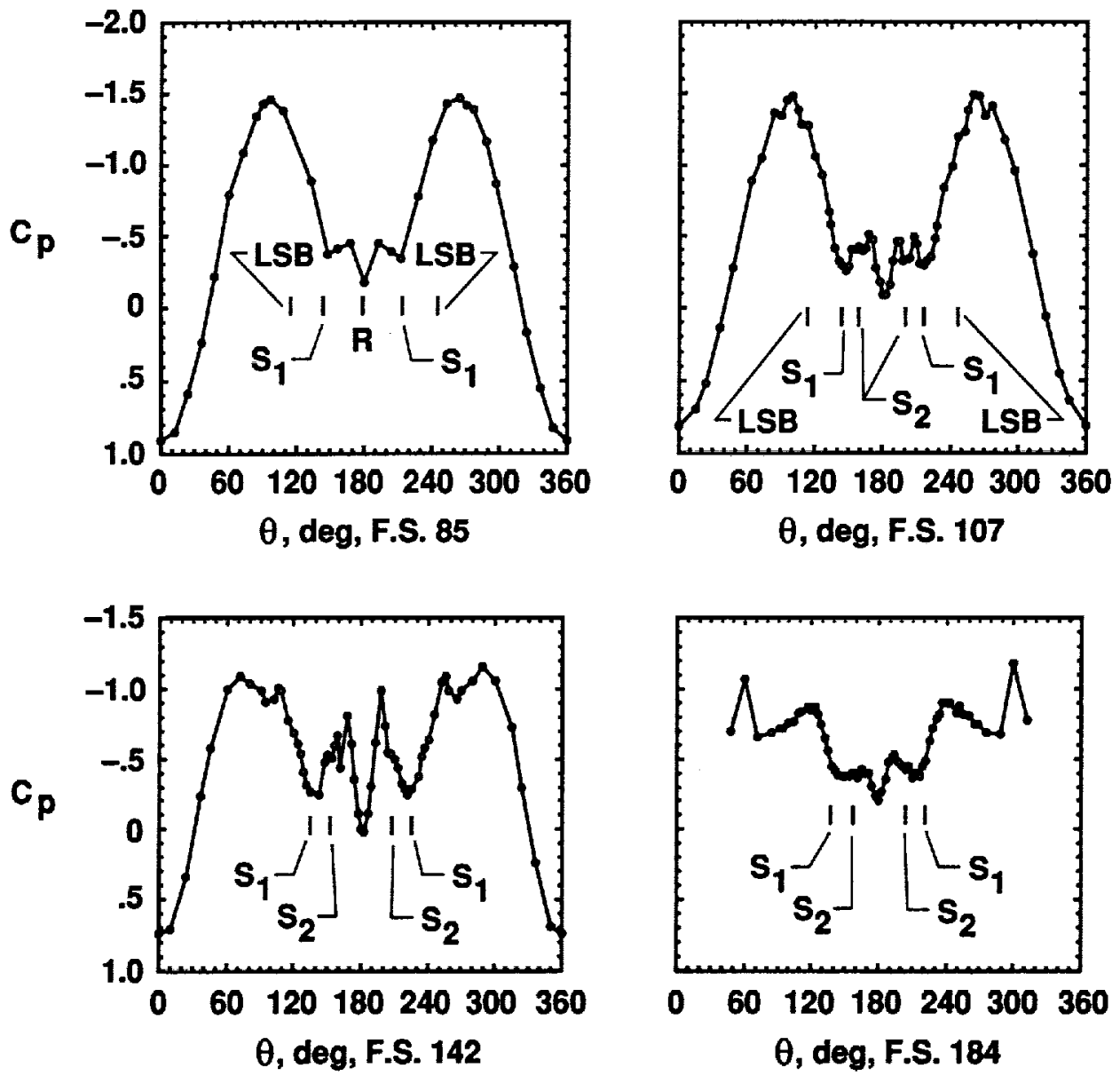

(b) $\alpha=48.1^{\circ} ; M_{\infty}=0.23$.

Fig. 10 Concluded. 

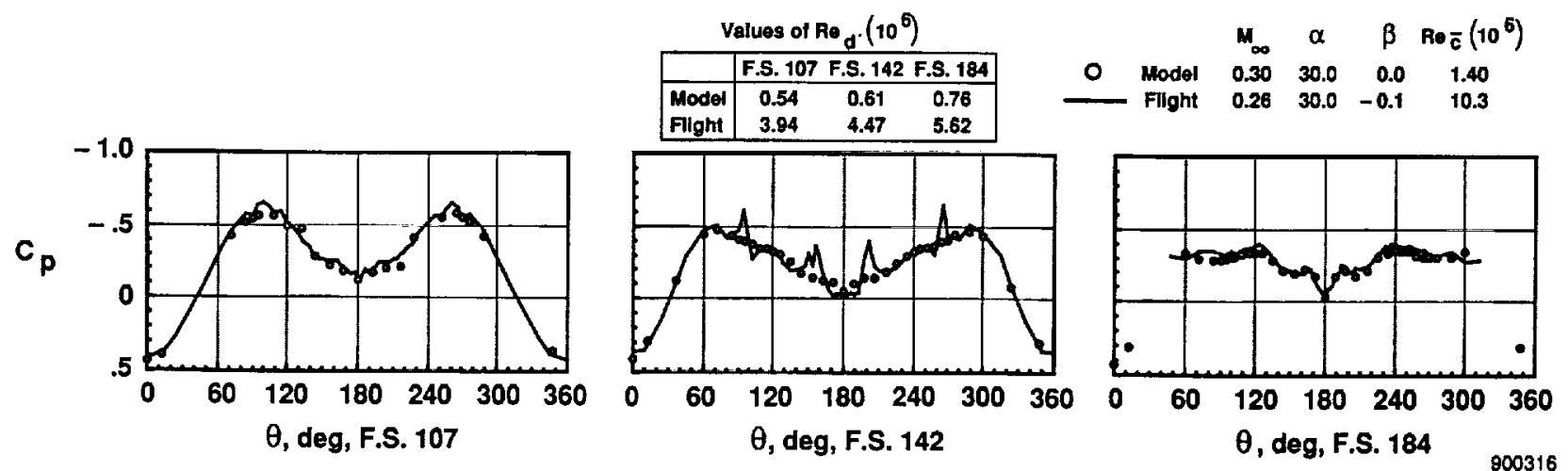

(a) $\alpha=30.0^{\circ} ; M_{\infty} \sim 0.30$.
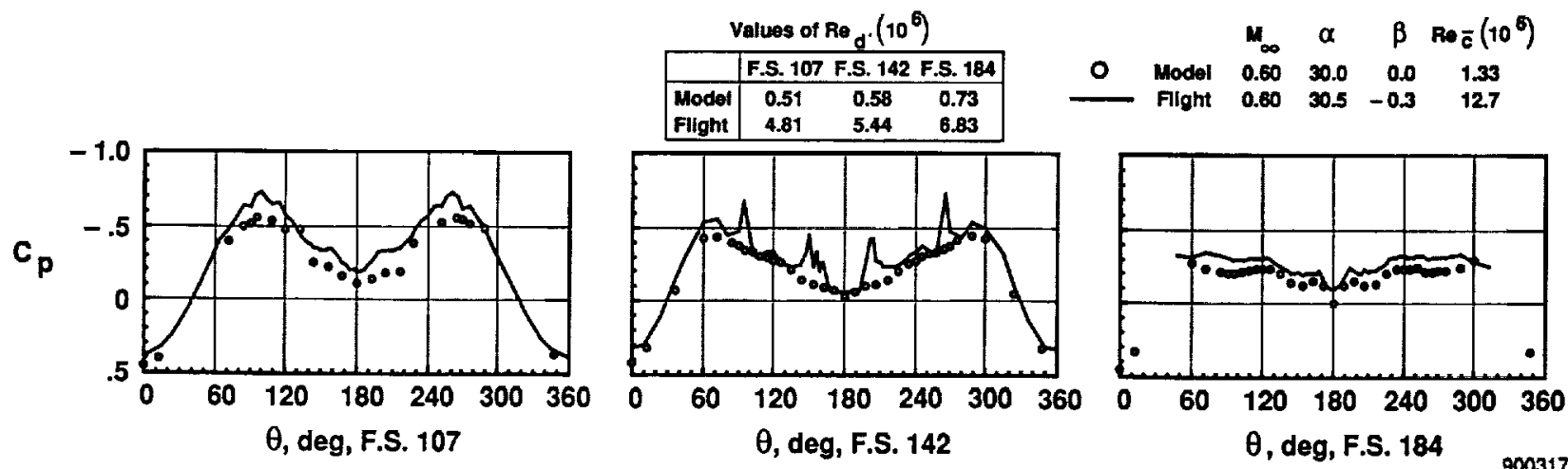

(b) $\alpha \sim 30^{\circ} ; M_{\infty}=0.60$.
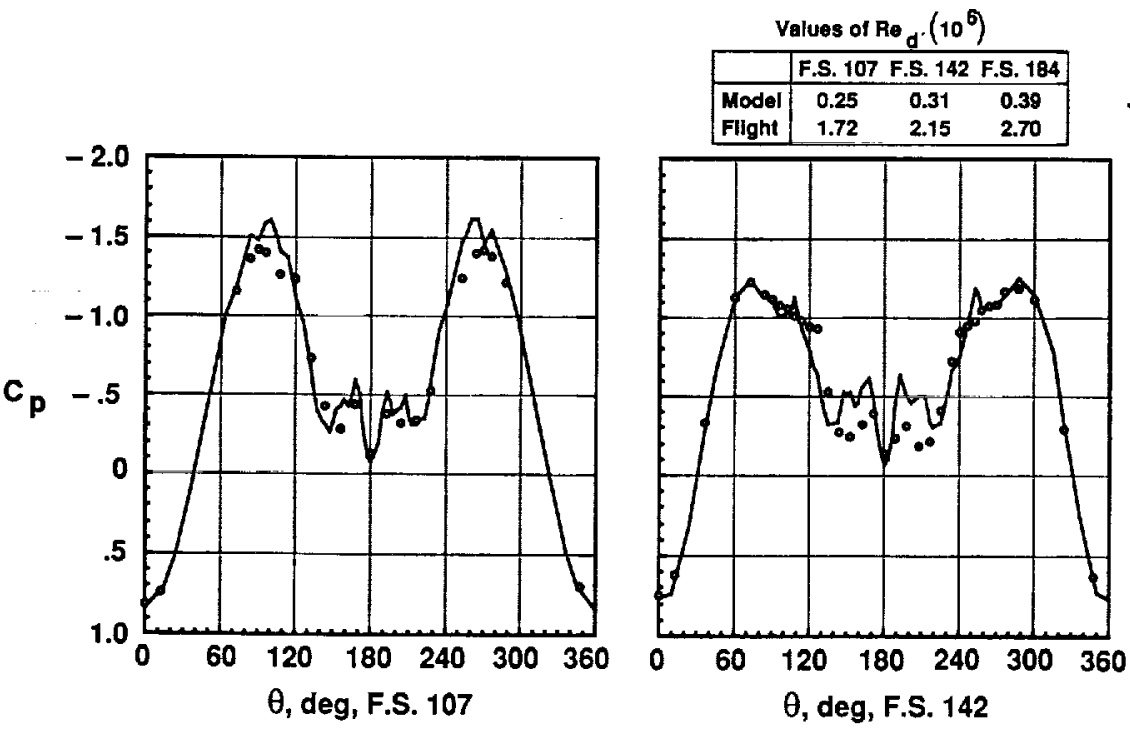

(c) $\alpha=50.0^{\circ} ; M_{\infty} \sim 0.20$.
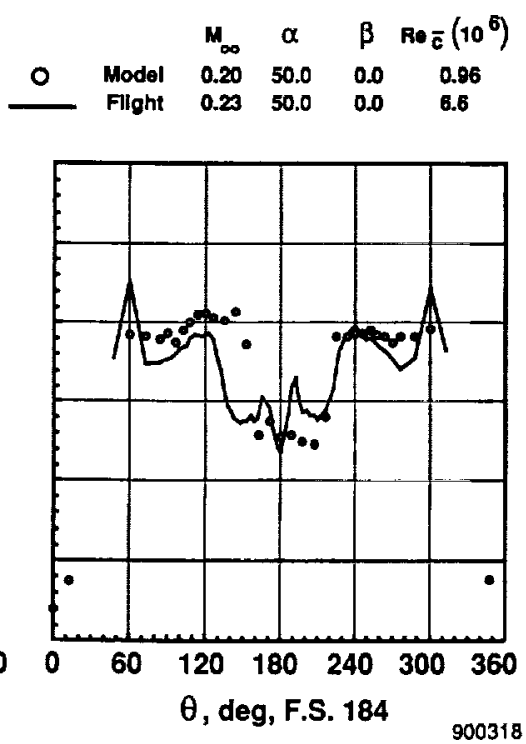

Fig. 11 Comparison of flight- and wind-tunnel-measured surface static pressure cocfficients on the F-18 HARV forcbody. 


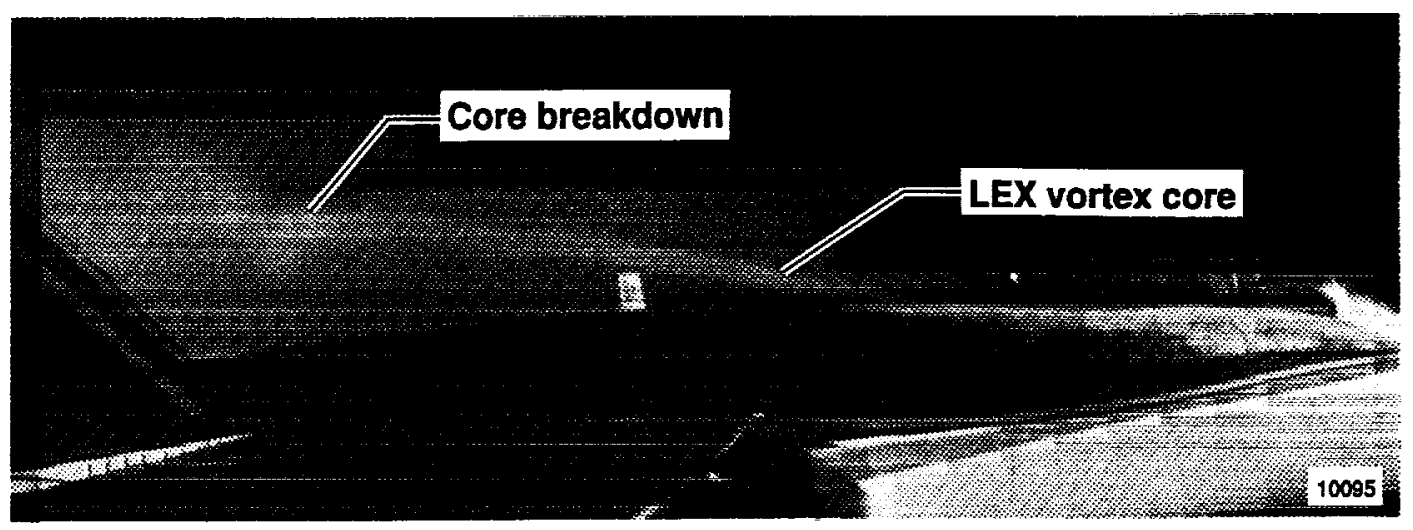

(a) $\alpha=20.0^{\circ}$ and $\beta=0^{\circ}$.

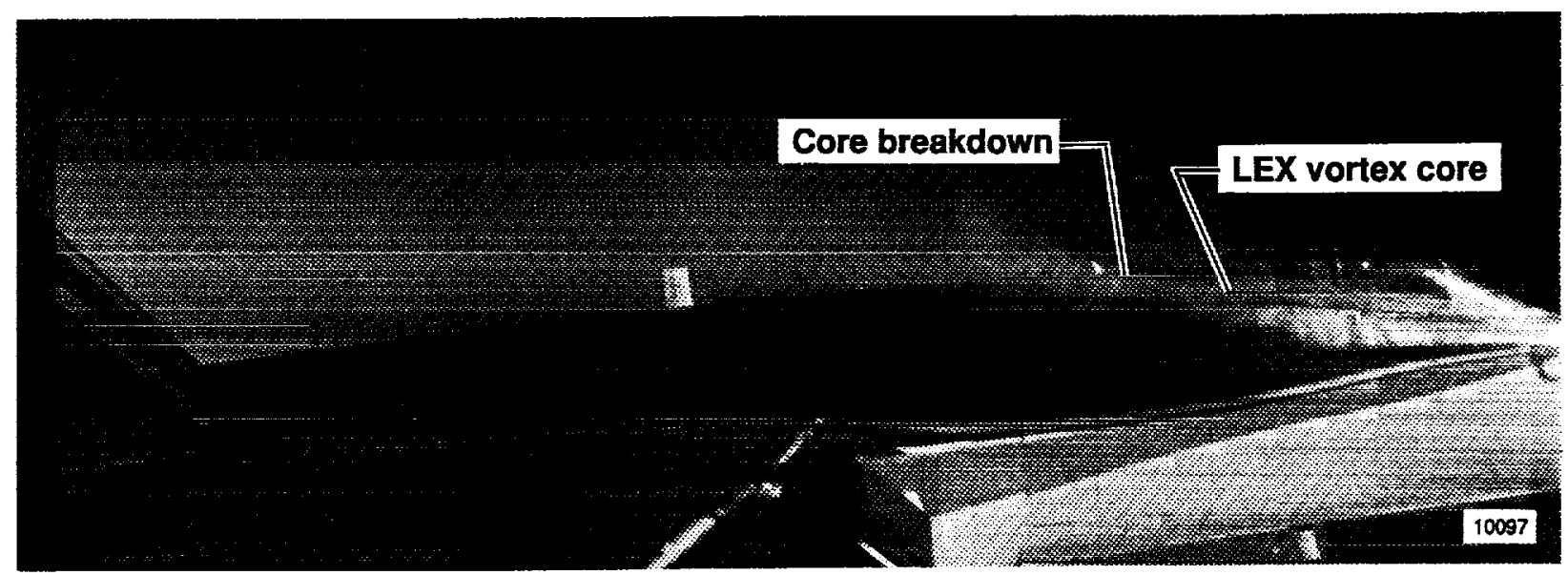

(b) $\alpha=29.8^{\circ}$ and $\beta=0.2^{\circ}$.

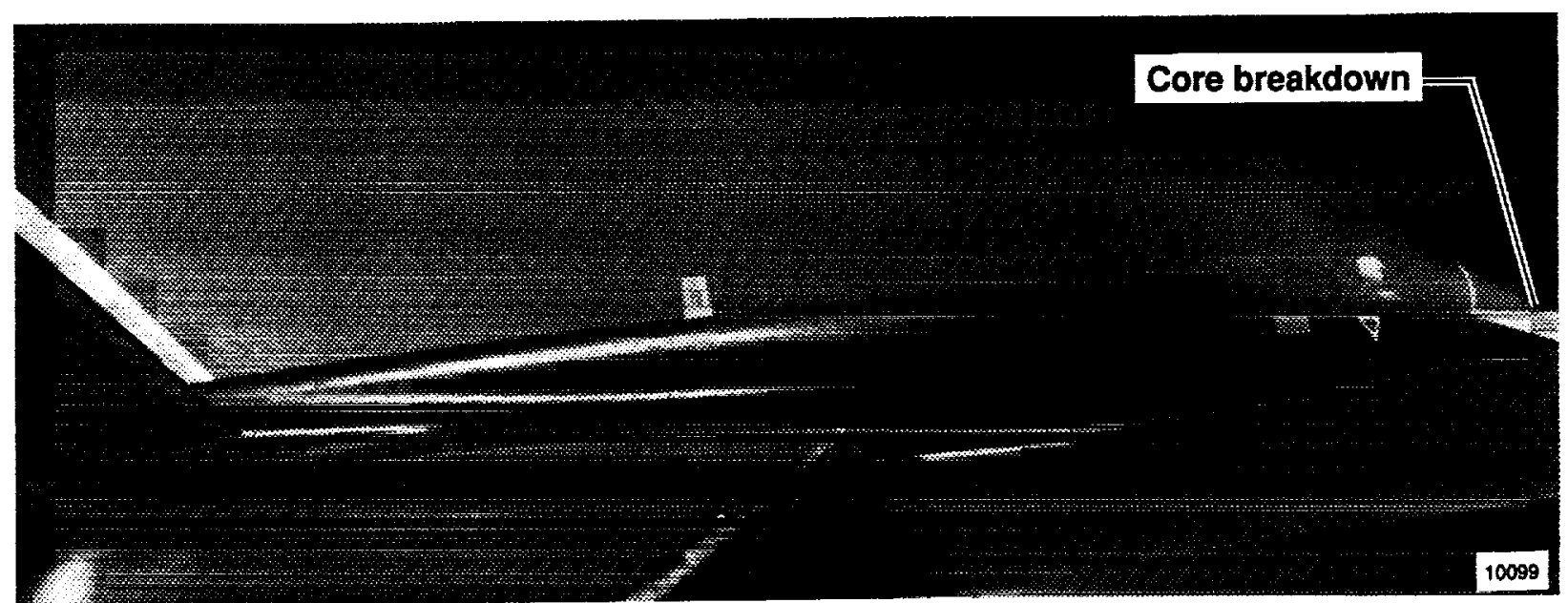

(c) $\alpha=42.5^{\circ}$ and $\beta=0.8^{\circ}$

Fig. 12 Wingtip view of smoke flow visualization on F-18 HARV LEX. 

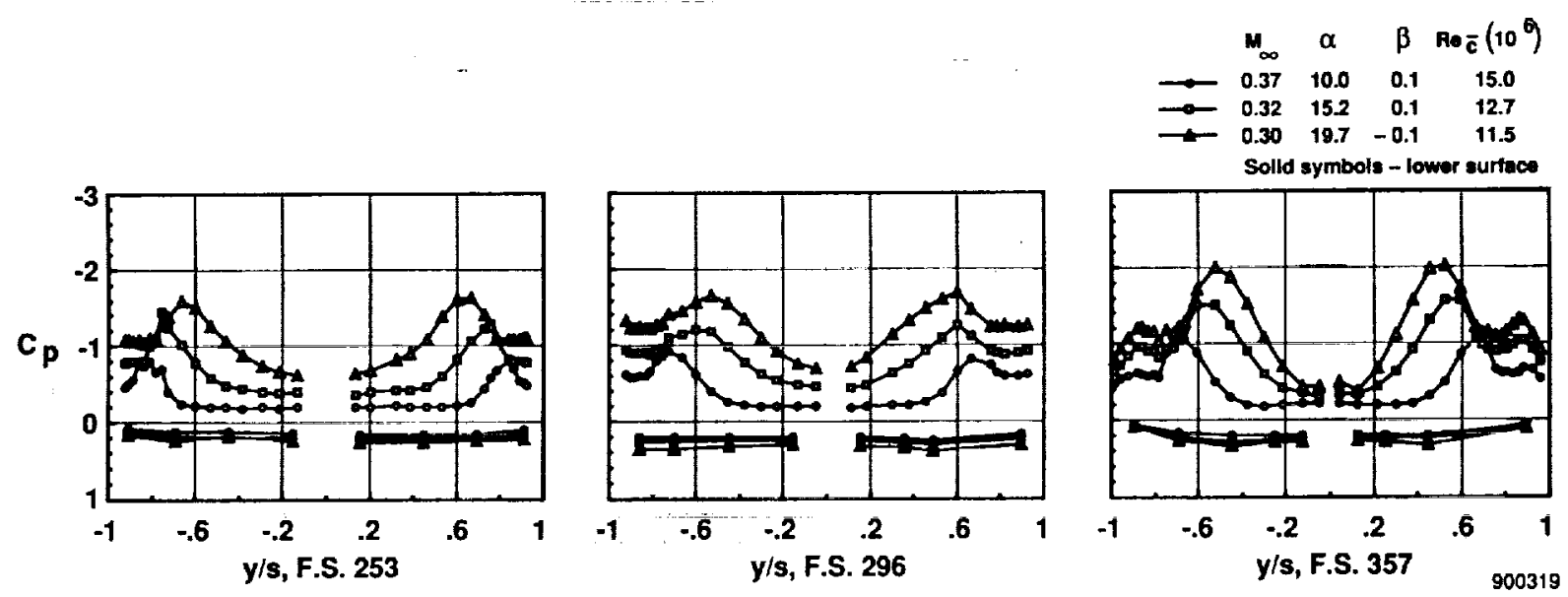

(a) $\alpha=10.0^{\circ}, 15.2^{\circ}$, and $19.7^{\circ} ; M_{\infty} \sim 0.35$.
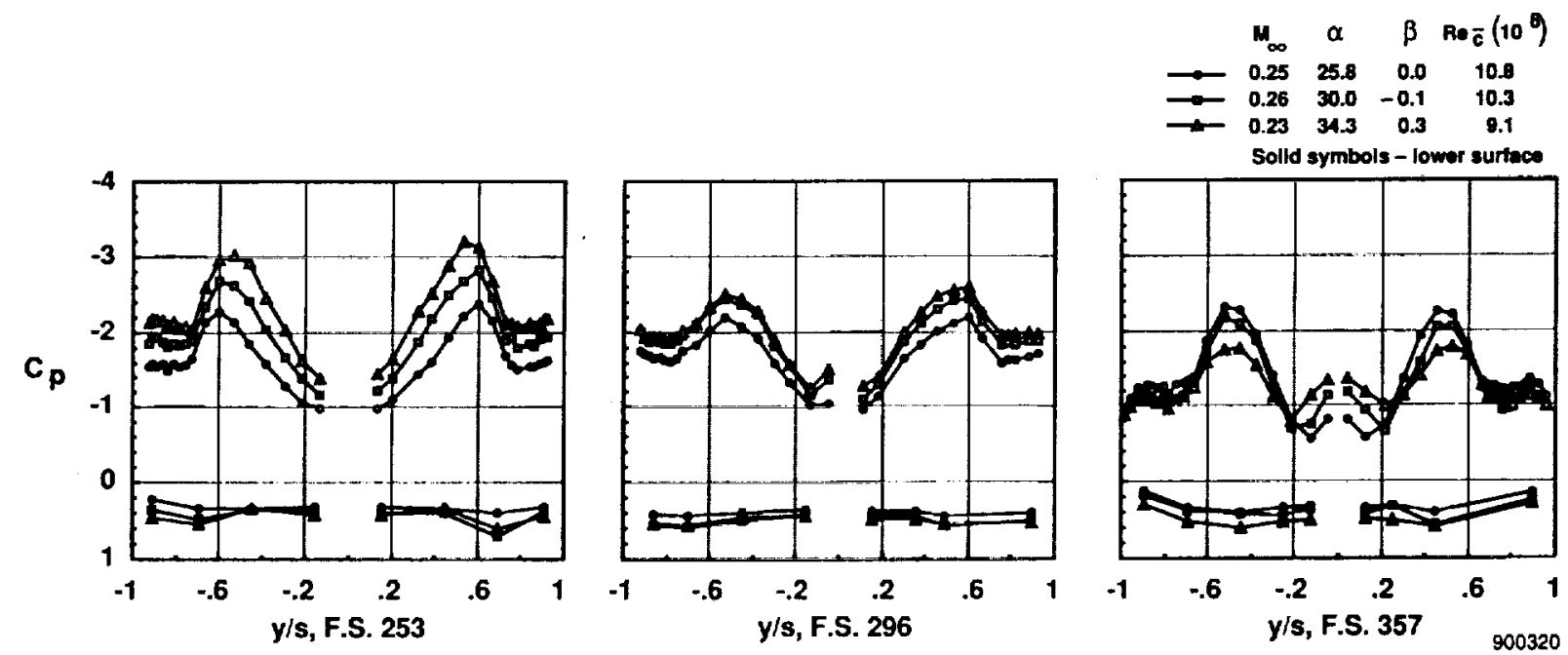

(b) $\alpha=25.8^{\circ}, 30.0^{\circ}$, and $34.3^{\circ} ; M_{\infty} \sim 0.25$.
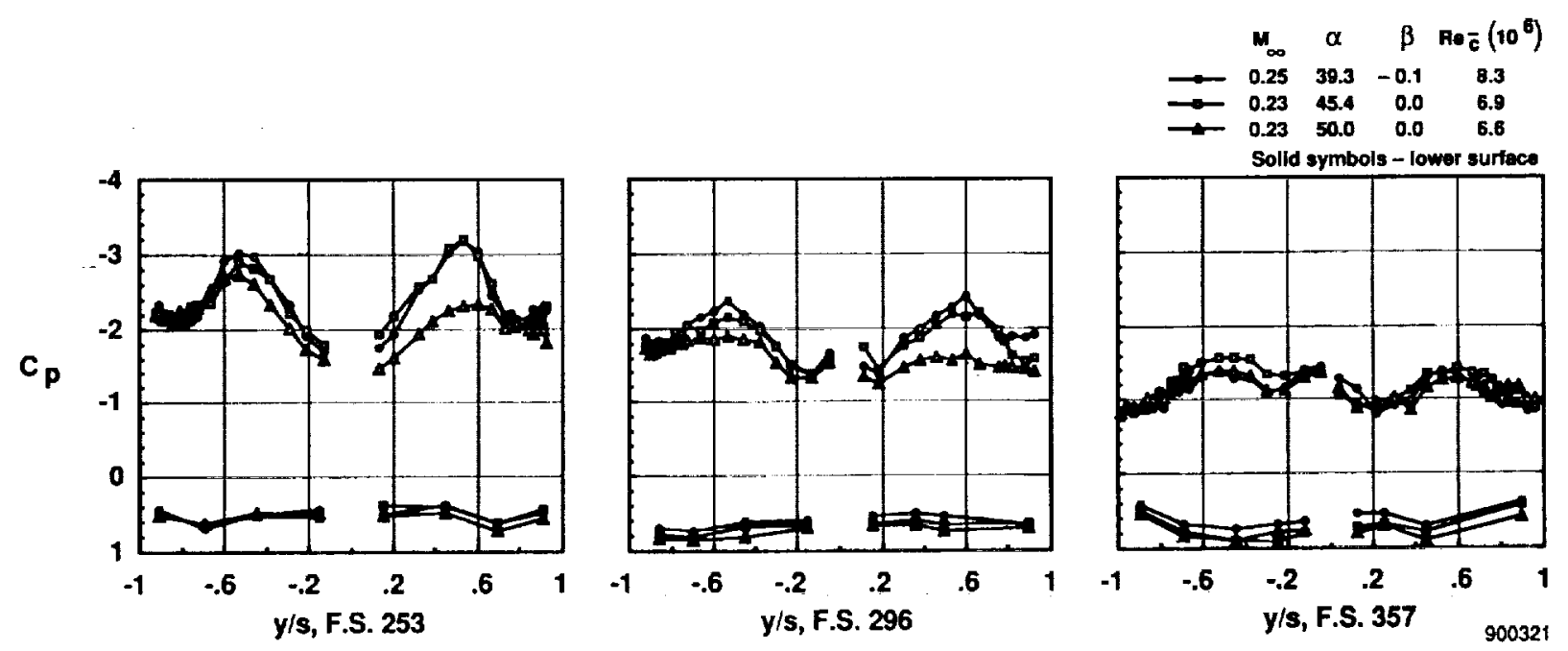

(c) $\alpha=39.4^{\circ}, 45.4^{\circ}$, and $50.0^{\circ} ; M_{\infty} \sim 0.25$.

Fig. 13 Effect of angle of attack on LEX surface static pressure cocfficients on the F-18 HARV at low specd. 


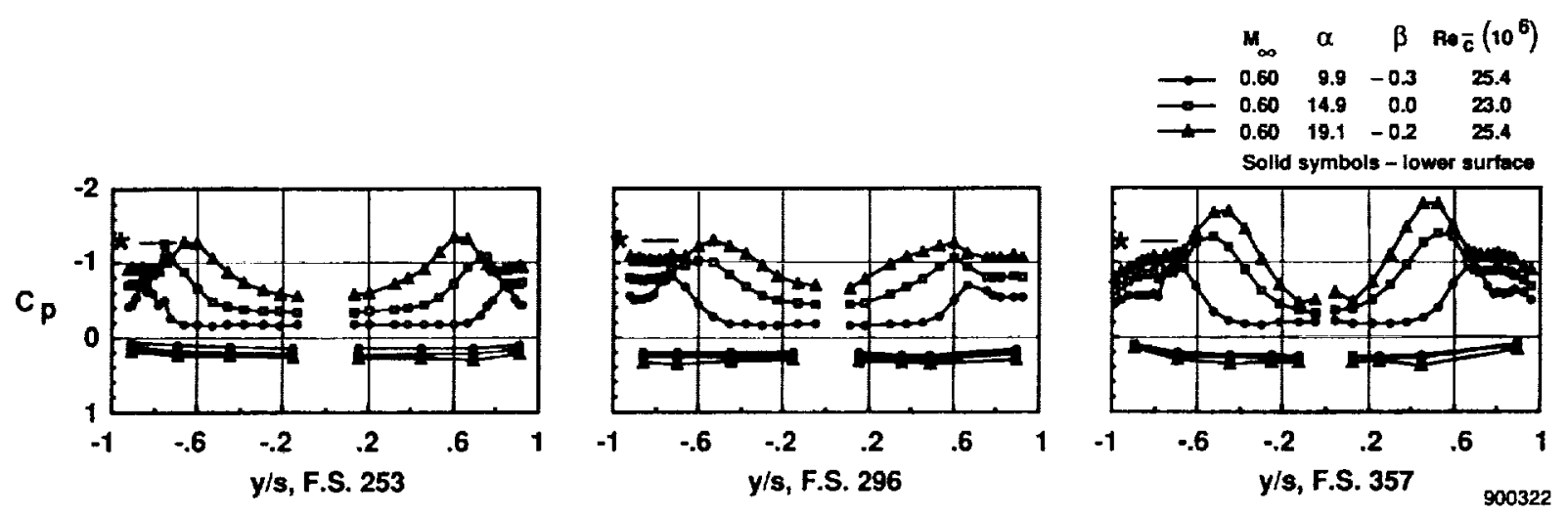

(a) $\alpha=9.9^{\circ}, 14.9^{\circ}$, and $19.1^{\circ}$.
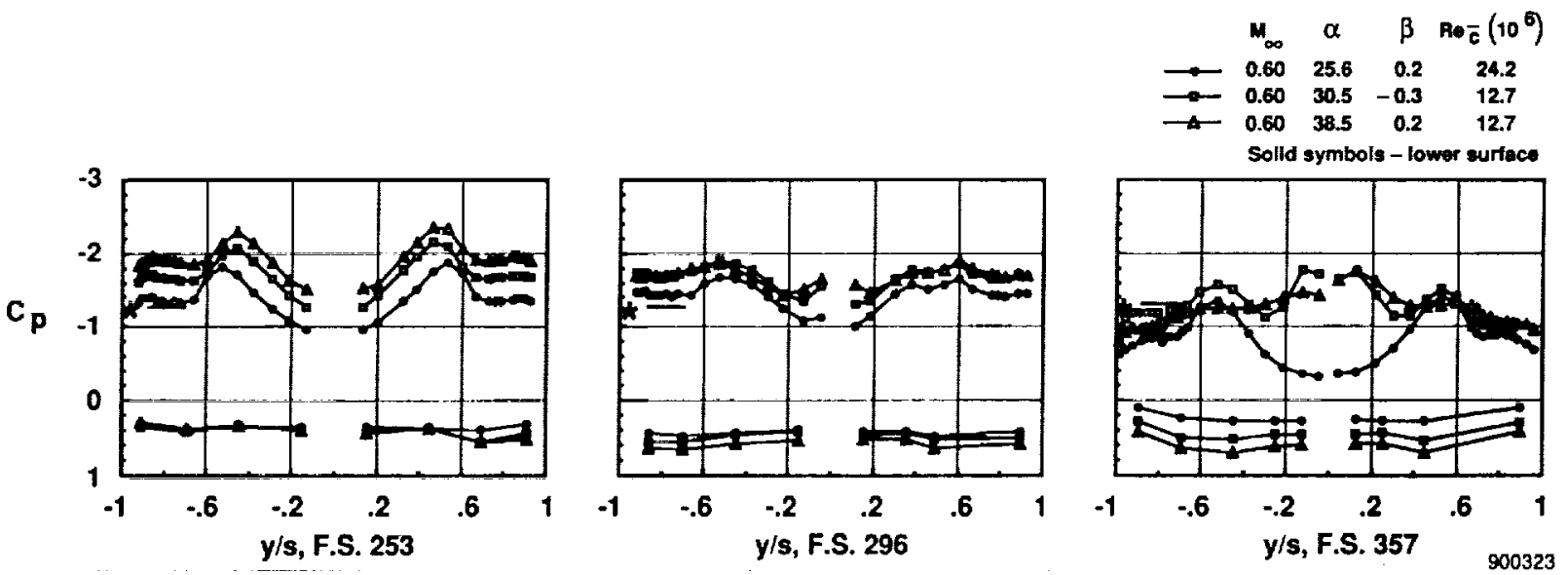

(b) $\alpha=25.6^{\circ}, 30.5^{\circ}$, and $38.5^{\circ}$.

Fig. 14 Effect of angle of attack on LEX surface slatic pressurc coefficients on the F-18 HARV at $M_{\infty} \sim 0.60$.
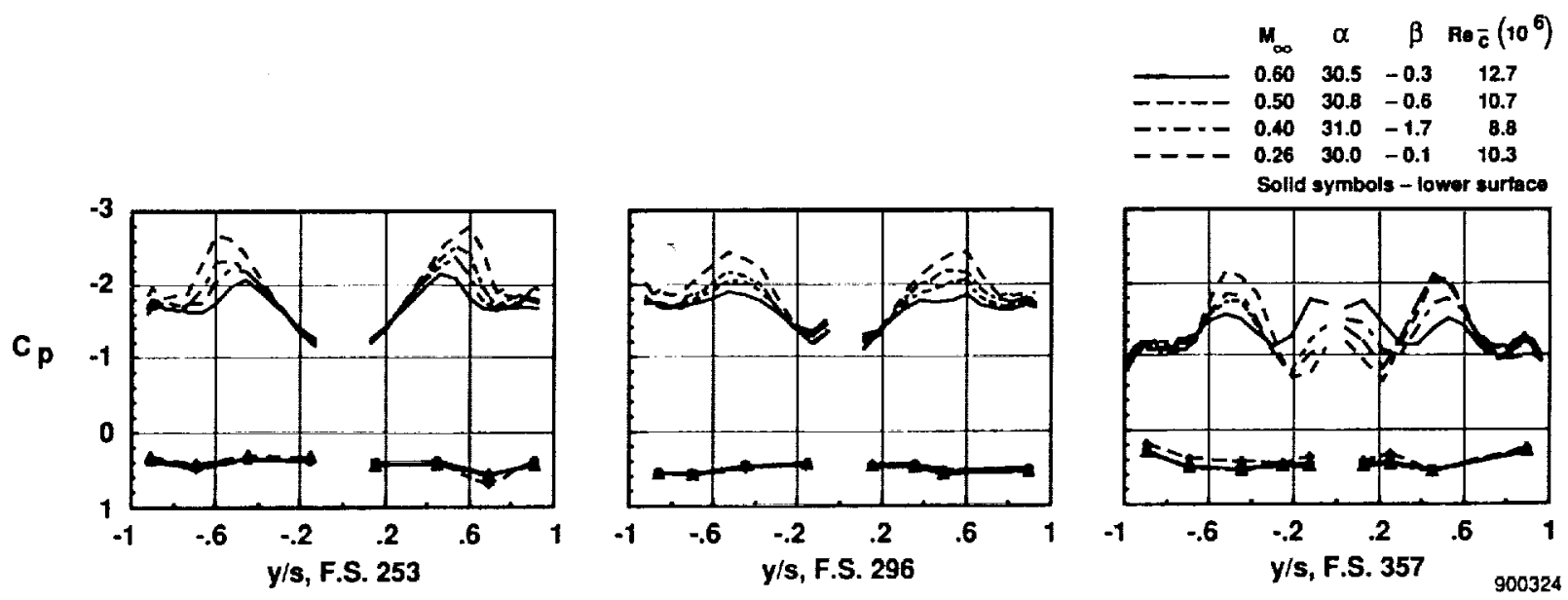

Fig. 15 Effect of Mach number on LEX surface static pressure cocfficients on the F-18 HARV at $\alpha \sim 31.0^{\circ}$. 


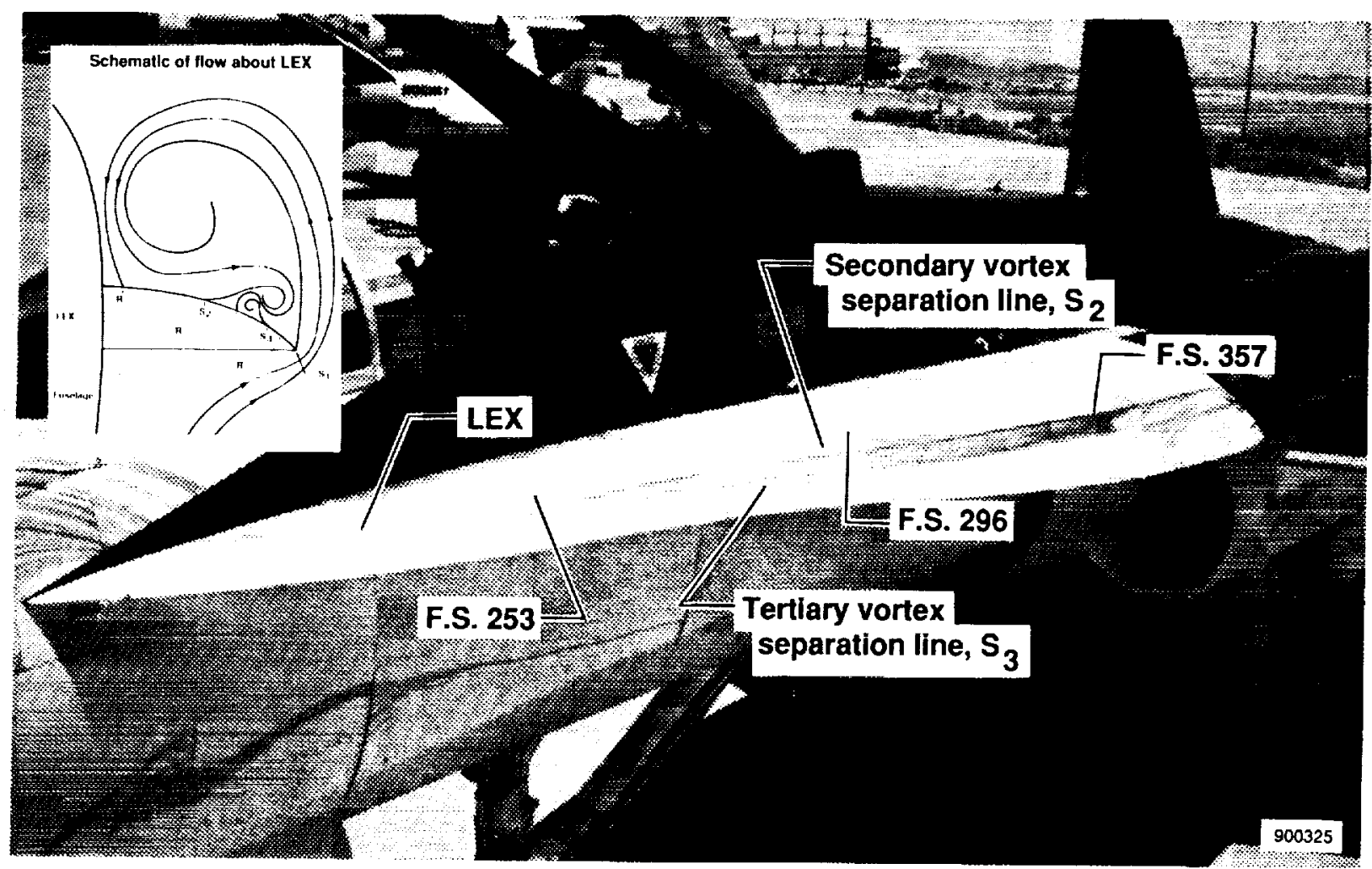

(a) $\alpha \sim 30^{\circ}$.

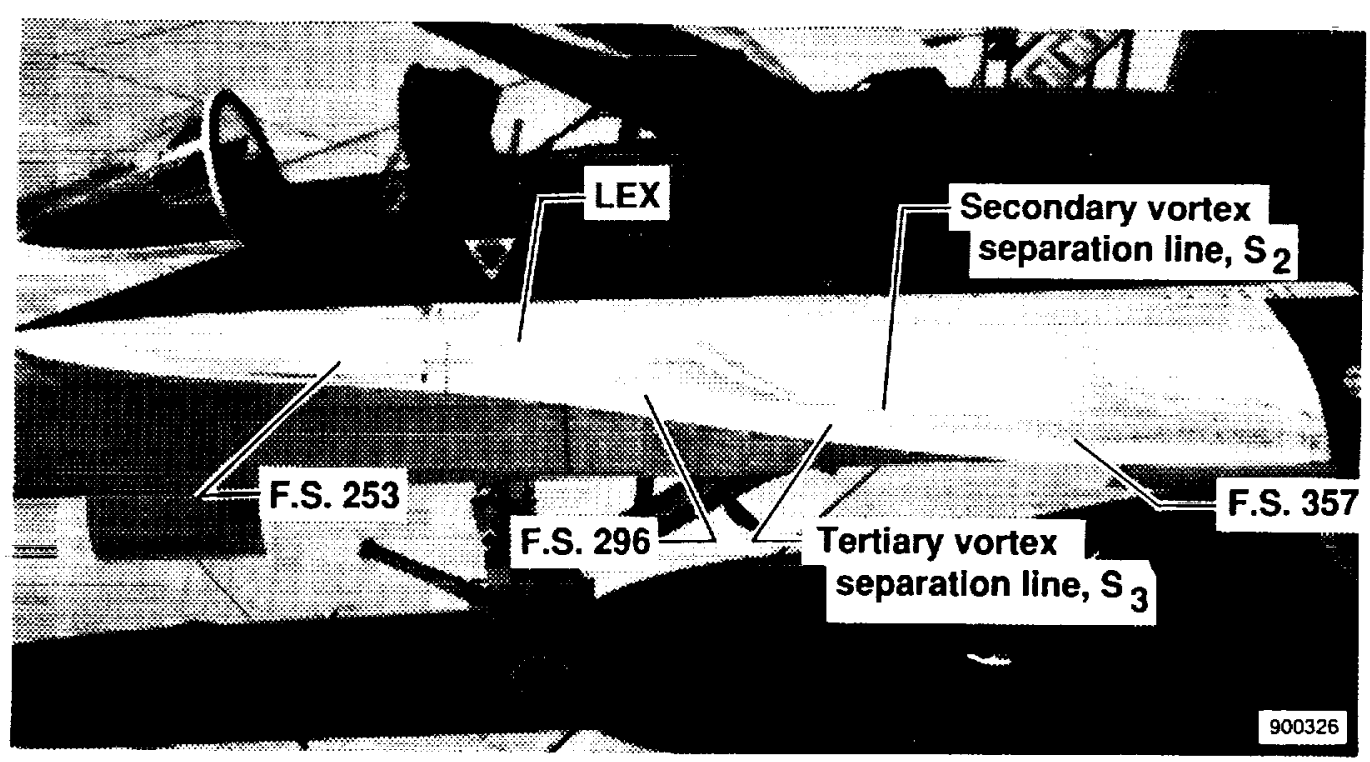

(b) $\alpha \sim 47^{\circ}$.

Fig. 16 Surface flow visualization on Ieft LEX of F-18 HARV. 

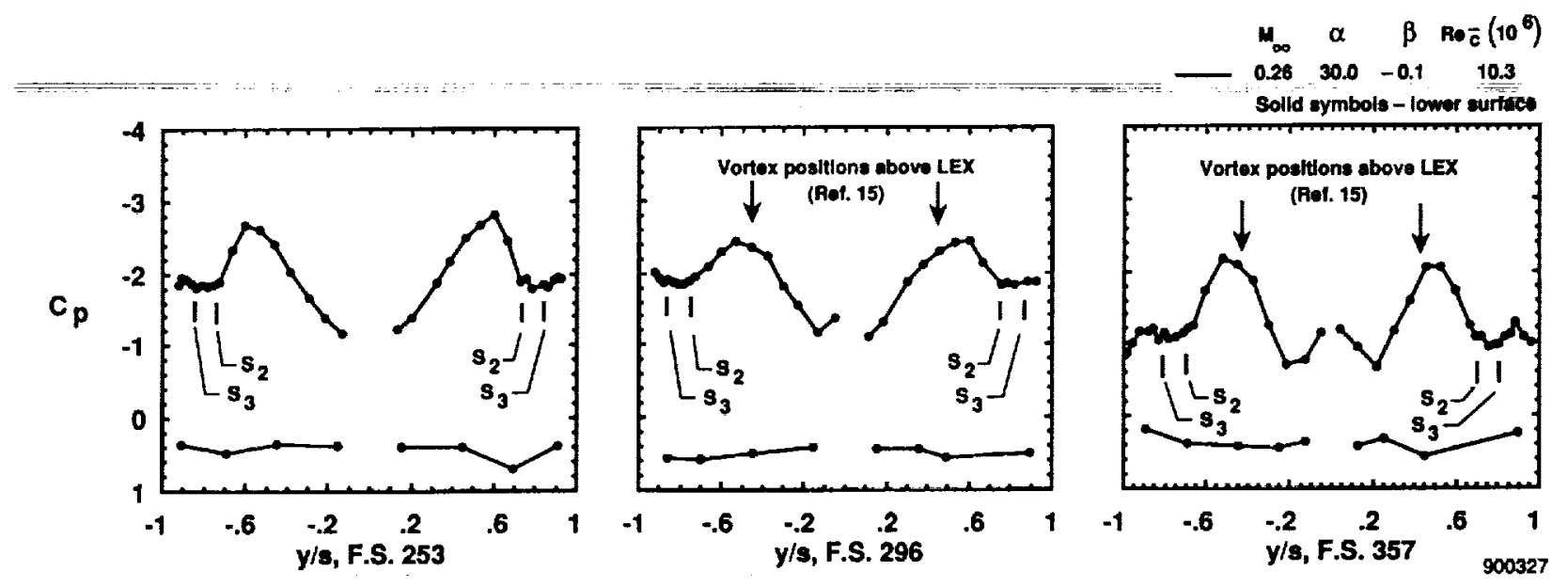

(a) $\alpha=30.0^{\circ} ; M_{\infty}=0.26$.
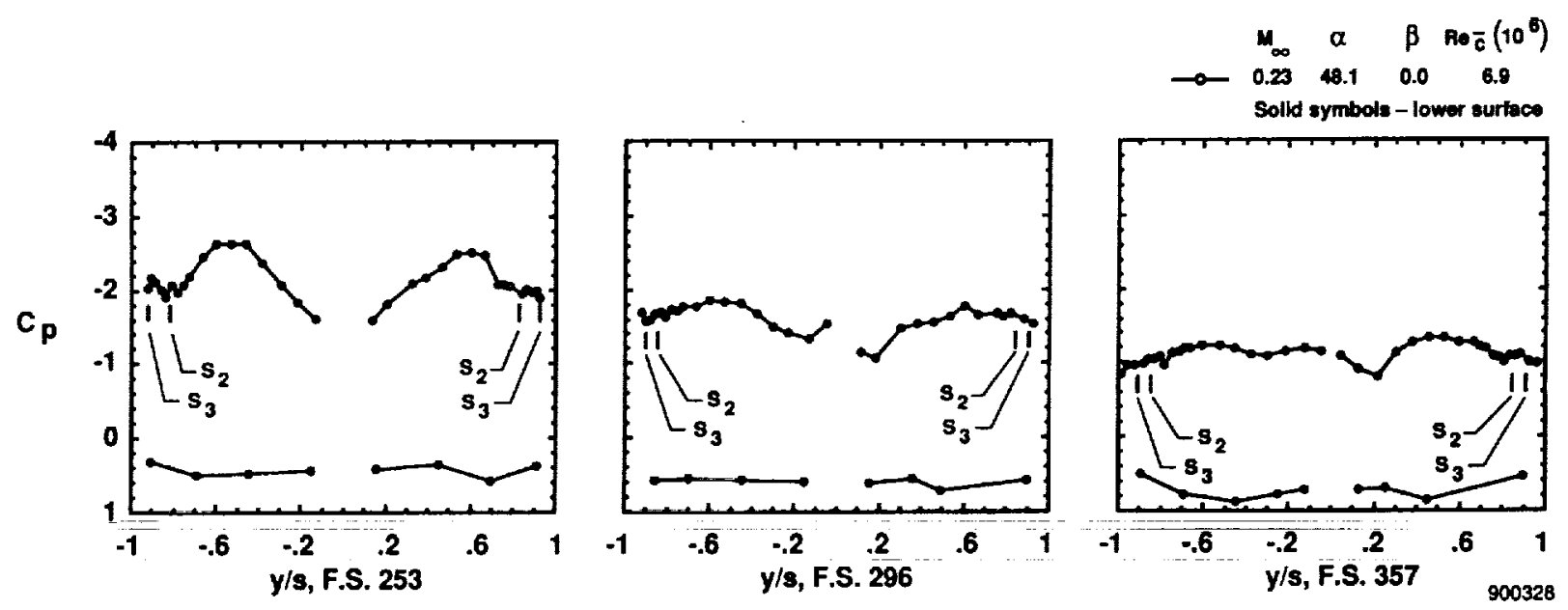

(b) $\alpha=48.1^{\circ} ; M_{\infty}=0.23$.

Fig. 17 Comparison of LEX surface static pressure coefficients with flow visualization results on the F-18 HARV. 

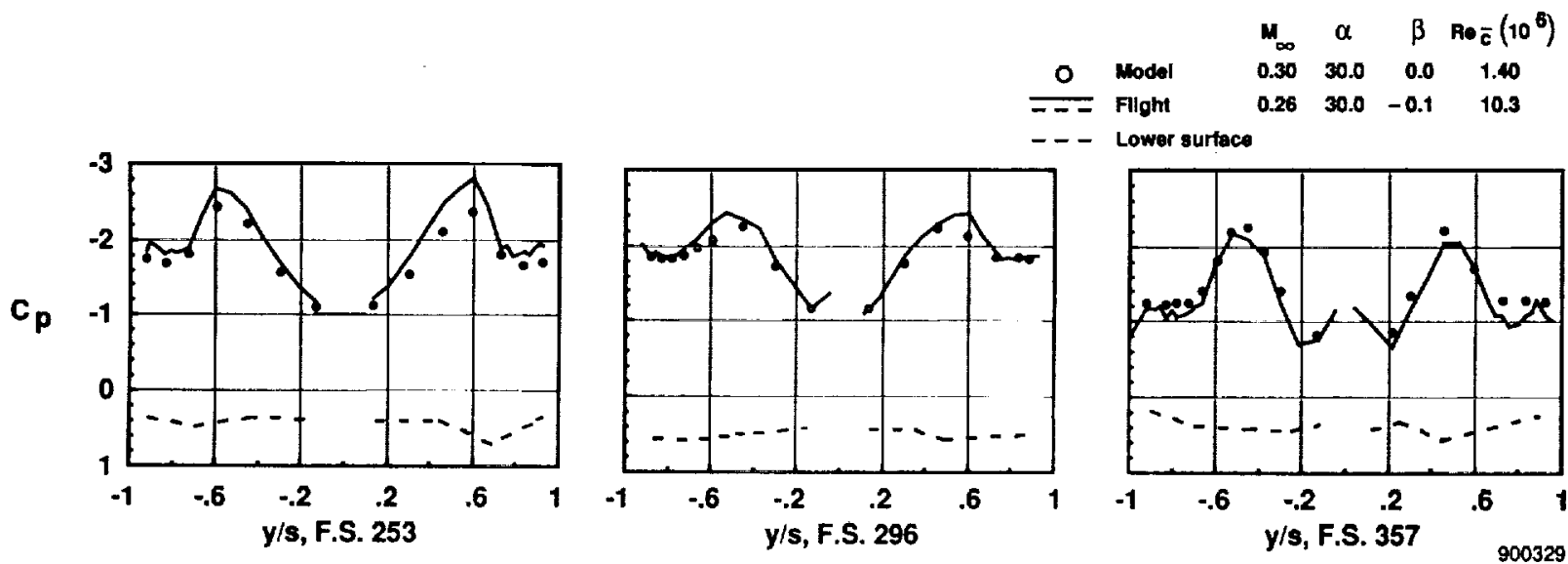

(a) $\alpha=30.0^{\circ} ; M_{\infty} \sim 0.30$.
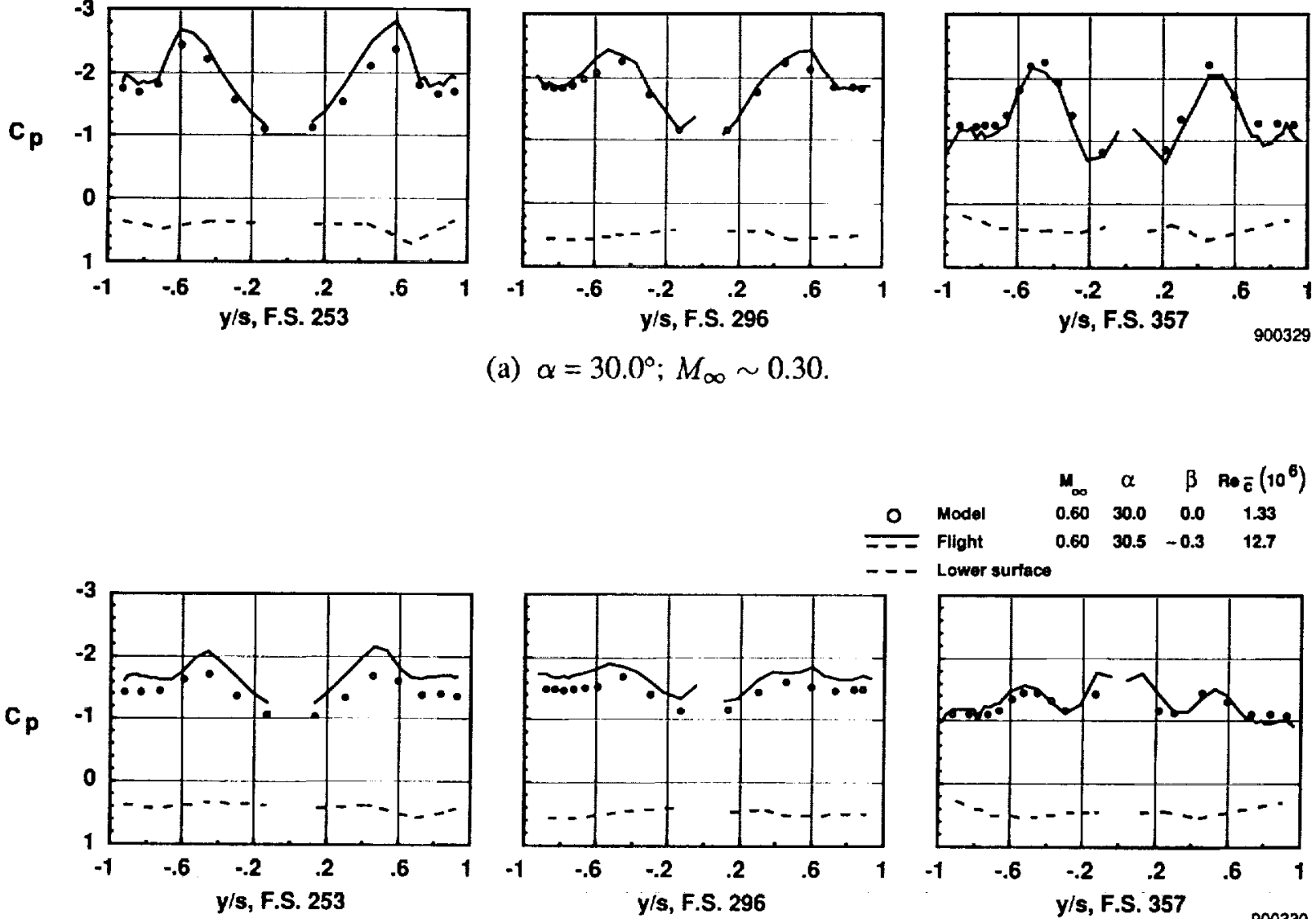

(b) $\alpha \sim 30^{\circ} ; M_{\infty}=0.60$.
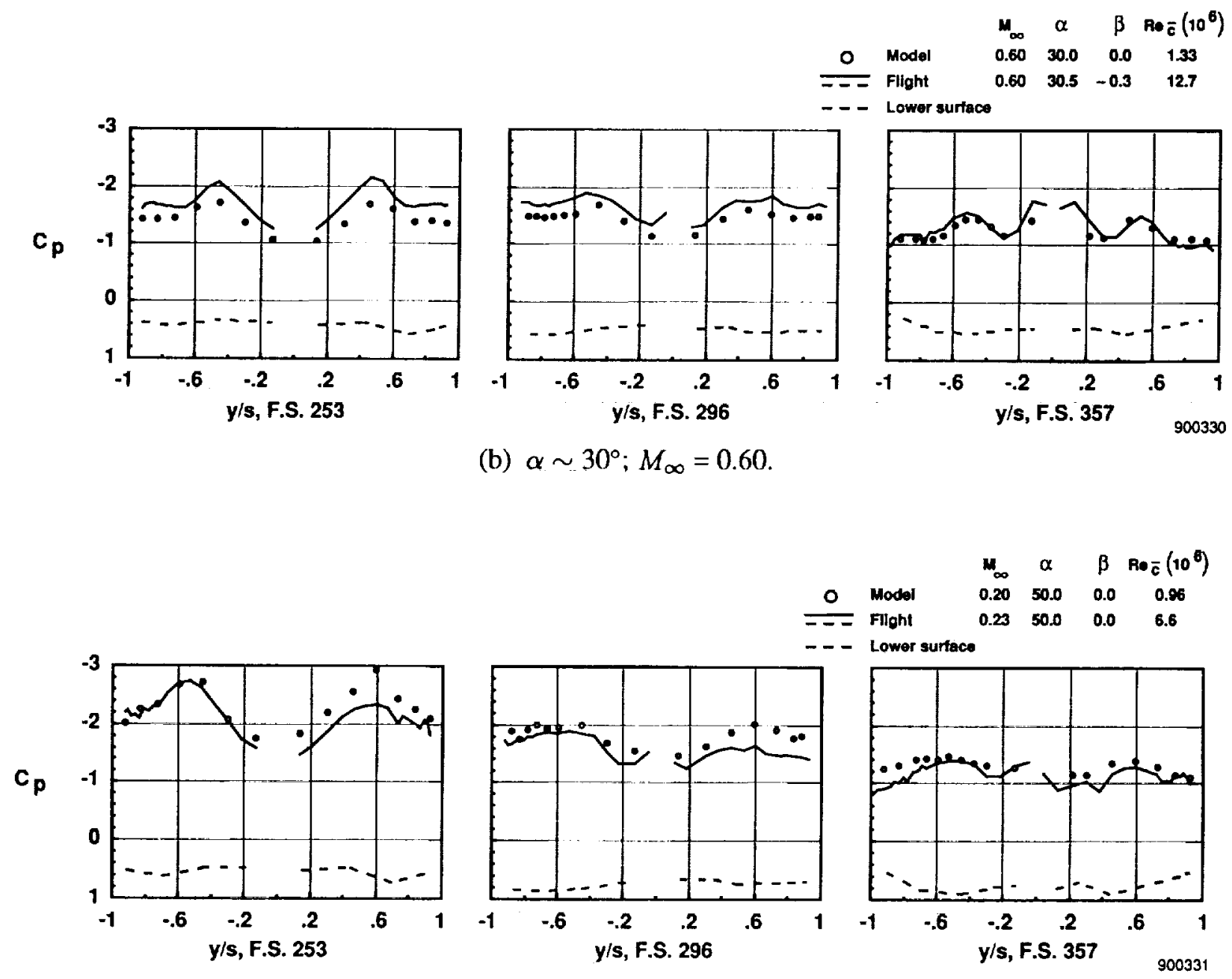

(c) $\alpha=50.0^{\circ} ; M_{\infty} \sim 0.23$.

Fig. 18 Comparison of flight- and wind-tunnel-measured LEX surface static pressure coefficients on the F-18. 


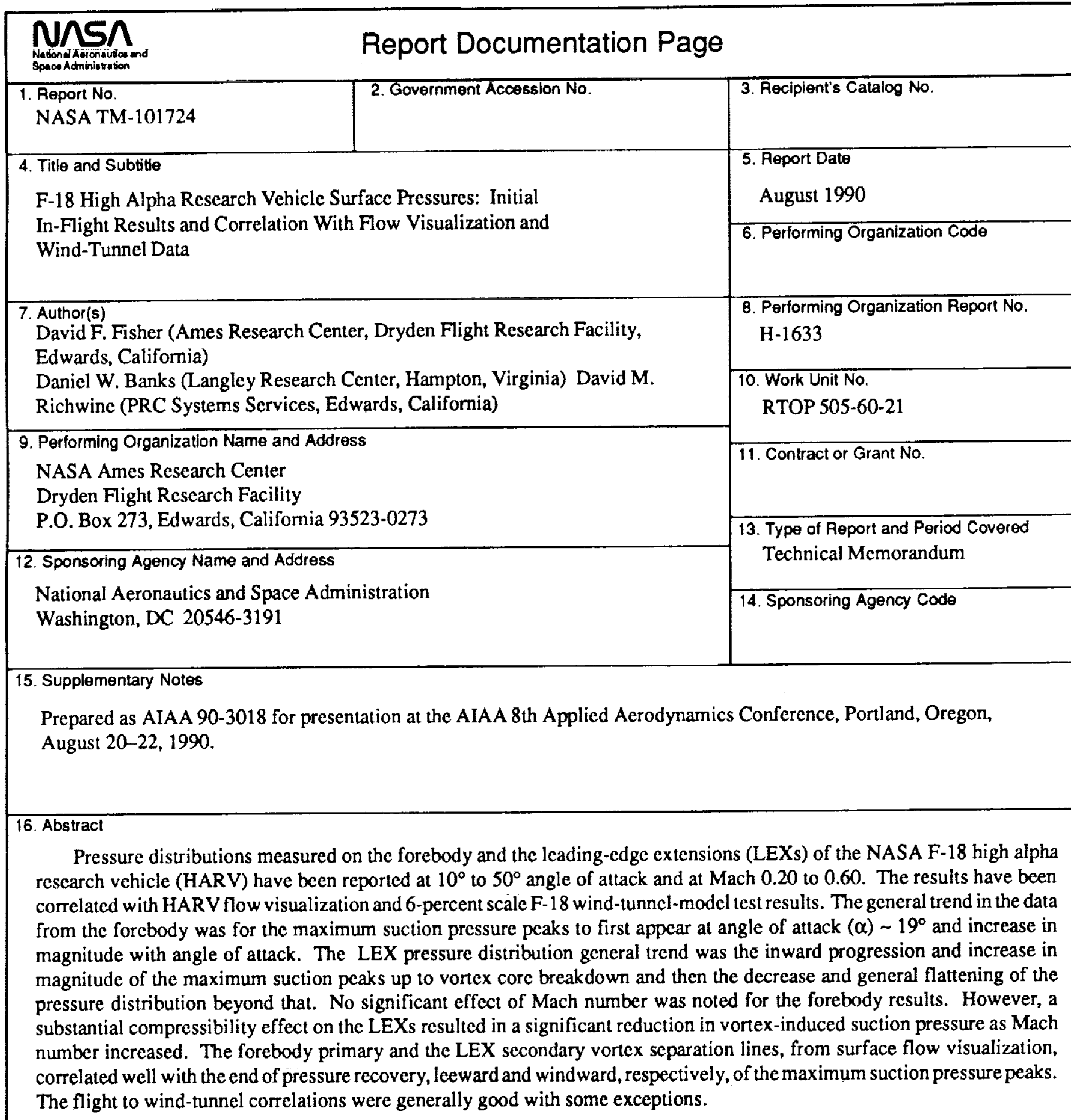

